\title{
Accessible lepton-number-violating models and negligible neutrino masses
}

\author{
André de Gouvêa $\oplus^{1,{ }^{*}}$ Wei-Chih Huang, ${ }^{2, \dagger}$ Johannes König, ${ }^{2, *}$ and Manibrata Sen $\oplus^{1,3, \S}$ \\ ${ }^{1}$ Northwestern University, Department of Physics and Astronomy, \\ 2145 Sheridan Road, Evanston, Illinois 60208, USA \\ ${ }^{2} C P^{3}$-Origins, University of Southern Denmark, Campusvej 55, DK-5230 Odense M, Denmark \\ ${ }^{3}$ Department of Physics, University of California Berkeley, Berkeley, California 94720, USA
}

(Received 18 July 2019; published 25 October 2019)

\begin{abstract}
Lepton-number violation (LNV), in general, implies nonzero Majorana masses for the Standard Model neutrinos. Since neutrino masses are very small, for generic candidate models of the physics responsible for LNV, the rates for almost all experimentally accessible LNV observables - except for neutrinoless doublebeta decay - are expected to be exceedingly small. Guided by effective-operator considerations of LNV phenomena, we identify a complete family of models where lepton number is violated but the generated Majorana neutrino masses are tiny, even if the new-physics scale is below $1 \mathrm{TeV}$. We explore the phenomenology of these models, including charged-lepton flavor-violating phenomena and baryonnumber-violating phenomena, identifying scenarios where the allowed rates for $\mu^{-} \rightarrow e^{+}$-conversion in nuclei are potentially accessible to next-generation experiments.
\end{abstract}

DOI: 10.1103/PhysRevD.100.075033

\section{INTRODUCTION}

Lepton number and baryon number are, at the classical level, accidental global symmetries of the renormalizable Standard Model (SM) Lagrangian. ${ }^{1}$ If one allows for generic nonrenormalizable operators consistent with the SM gauge symmetries and particle content, lepton number and baryon number will no longer be conserved. Indeed, lepton-number conservation is violated by effective operators of dimension five or higher while baryon-number conservation (sometimes together with lepton number) is violated by effective operators of dimension six or higher. In other words, generically, the addition of new degrees-offreedom to the SM particle content violates baryon-number and lepton-number conservation.

\footnotetext{
*degouvea@northwestern.edu

†huang@cp3.sdu.dk

konig@cp3.sdu.dk

\$manibrata@berkeley.edu

${ }^{1}$ At the quantum level these symmetries are anomalous, i.e., they are violated by nonperturbative effects $[1,2]$. These are only relevant in extraordinary circumstances (e.g., very high temperatures) much beyond the reach of particle physics experiments $[3,4]$. Nonperturbative effects still preserve baryon-numberminus-lepton number, the nonanomalous possible global symmetry of the renormalizable SM Lagrangian.

Published by the American Physical Society under the terms of the Creative Commons Attribution 4.0 International license. Further distribution of this work must maintain attribution to the author(s) and the published article's title, journal citation, and DOI. Funded by SCOAP ${ }^{3}$.
}

Experimentally, in spite of ambitious ongoing experimental efforts, there is no evidence for the violation of lepton-number or baryon-number conservation [5]. There are a few different potential explanations for these (negative) experimental results, assuming degrees-of-freedom beyond those of the SM exist. Perhaps the new particles are either very heavy or very weakly coupled in such a way that phenomena that violate lepton-number or baryon-number conservation are highly suppressed. Another possibility is that the new interactions are not generic and that leptonnumber or baryon-number conservation are global symmetries of the beyond-the-Standard-Model Lagrangian. Finally, it is possible that even though baryon number or lepton number are not conserved and the new degrees-offreedom are neither weakly coupled nor very heavy, only a subset of baryon-number-violating or lepton-numberviolating phenomena are within reach of particle physics experiments. This manuscript concentrates on this third option, which we hope to elucidate below.

The discovery of nonzero yet tiny neutrino masses is often interpreted as enticing - but certainly not definitiveindirect evidence for lepton-number-violating new physics. In this case, neutrinos are massive Majorana fermions and one can naturally "explain" why the masses of neutrinos are much smaller than those of all other known massive particles (see, e.g., [6-8] for discussions of this point). Searches for the nature of the neutrino-Majorana fermion versus Dirac fermion-are most often searches for lepton number violation (LNV). The observation of LNV implies, generically, that neutrinos are Majorana fermions [9], while Majorana neutrino masses imply nonzero rates for 
lepton-number-violating phenomena. The most powerful probes of LNV are searches for neutrinoless double-beta decay $(0 \nu \beta \beta$, see [10] for a review); several of these are ongoing, e.g., [11-13]. The growing excitement behind searches for $0 \nu \beta \beta$ is the fact that these are sensitive enough to detect LNV mediated by light Majorana neutrino exchange if the neutrino masses are above a fraction on an electronvolt. In many models that lead to Majorana neutrino masses, including, arguably, the simplest, most elegant, and best motivated ones, LNV phenomena are predominantly mediated by light Majorana neutrino exchange. Hence, we are approaching sensitivities to $0 \nu \beta \beta$ capable of providing nontrivial, robust information on the nature of the neutrino.

Other searches for LNV are, in general, not as sensitive as those for $0 \nu \beta \beta$. Here, we will highlight searches for $\mu^{-} \rightarrow e^{+}$-conversion in nuclei, for a couple of reasons. One is that, except for searches for $0 \nu \beta \beta$, searches for $\mu^{-} \rightarrow e^{+}$conversion in nuclei are, arguably, the most sensitive to generic LNV new physics. ${ }^{2}$ Second, several different experiments aimed at searching for $\mu^{-} \rightarrow e^{-}$-conversion in nuclei are under construction, including the COMET [15] and DeeMe experiments [16] in J-PARC, and the $M u 2 e$ experiment [17] in Fermilab. These efforts are expected to increase the sensitivity to $\mu^{-} \rightarrow e^{-}$-conversion by, ultimately, four orders of magnitude and may also be able to extend the sensitivity to $\mu^{-} \rightarrow e^{+}$-conversion in nuclei by at least a few orders of magnitude.

The best bounds on the $\mu^{-} \rightarrow e^{+}$-conversion rate relative to the capture rate of a $\mu^{-}$on titanium were obtained by the SINDRUM II experiment [18] over twenty years ago:

$$
\begin{aligned}
R_{\mu^{-} e^{+}}^{\mathrm{Ti}} & \equiv \frac{\Gamma\left(\mu^{-}+\mathrm{Ti} \rightarrow e^{+}+\mathrm{Ca}\right)}{\Gamma\left(\mu^{-}+\mathrm{Ti} \rightarrow \nu_{\mu}+\mathrm{Sc}\right)} \\
& < \begin{cases}1.7 \times 10^{-12} & (\mathrm{GS}, 90 \% \mathrm{CL}) \\
3.6 \times 10^{-11} & (\mathrm{GDR}, 90 \% \mathrm{CL})\end{cases}
\end{aligned}
$$

where GS considers scattering off titanium to the ground state of calcium, whereas GDR considers the transition to a giant dipole resonance (GDR) state. Next-generation experiments like $M u 2 e$, DeeMe, and COMET have the potential to be much more sensitive to $\mu^{-} \rightarrow e^{+}$-conversion. The authors of [19] naively estimated the future sensitivities of these experiments to be

$$
\mathrm{Mu} 2 \mathrm{e}: R_{\mu^{-} e^{+}}^{\mathrm{Al}} \gtrsim 10^{-16},
$$

\footnotetext{
${ }^{2}$ It was recently pointed out that searches for nonstandard neutrino interactions from long-baseline neutrino experiments are also sensitive to certain LNV new physics and involve all lepton flavors [14]. In some cases, the resulting limits are stronger than those from $\mu^{-} \rightarrow e^{+}$-conversion.
}

$$
\text { COMET Phase-I: } R_{\mu^{-} e^{+}}^{\mathrm{Al}} \gtrsim 10^{-14} \text {. }
$$

For a recent, more detailed discussion, see [20].

There are several recent phenomenological attempts at understanding whether there are models consistent with current experimental constraints where the rate for $\mu^{-} \rightarrow e^{+}$-conversion in nuclei is sizable $[19,21,22]$. The main challenges are two-fold. On the one hand, the lightMajorana-neutrino exchange contribution to $\mu^{-} \rightarrow e^{+}$conversion in nuclei is tiny. On the other hand, while it is possible to consider other LNV effects that are not captured by light-Majorana-neutrino exchange, most of these scenarios lead, once the new degrees of freedom are integrated out, to Majorana neutrino masses that are way too large and safely excluded by existing neutrino data. In [19], an effective operator approach, introduced and exploited in, e.g, [23-26], was employed to both diagnose the problem and identify potentially interesting directions for model building.

New-physics scenarios that violate lepton-number conservation at the tree level in a way that LNV low-energy phenomena are captured by the "all-singlets" dimensionnine operator:

$$
\mathcal{L} \supset \frac{1}{\Lambda^{5}} \mathcal{O}_{s}, \quad \text { where } \mathcal{O}_{s}=e^{c} \mu^{c} u^{c} u^{c} \overline{d^{c}} \overline{d^{c}},
$$

and $\Lambda$ is the effective scale of the operator, were flagged as very "inefficient" when it comes to generating neutrino Majorana masses. According to [19], the contribution to Majorana neutrino masses from the physics that leads to Eq. (1.4) at the tree level saturates the upper bound on neutrino masses for $\Lambda \sim 1 \mathrm{GeV}$. This means that, for $\Lambda \gg 1 \mathrm{GeV}$, the physics responsible for Eq. (1.4) will lead to neutrino masses that are too small to be significant while the rates of other LNV phenomena, including $\mu^{-} \rightarrow e^{+}$-conversion in nuclei, may be within reach of next-generation experiments. According to [19], this happens for $\Lambda \lesssim 100 \mathrm{GeV}$.

The effective operator approach from [23-26] is mostly powerless when it comes to addressing lepton-numberconserving, low-energy effects of the same physics that leads to Eq. (1.4). One way to understand this is to appreciate that lepton-number-conserving phenomena are captured by qualitatively different effective operators and, in general, it is not possible to relate different "types" of operators in a model-independent way. Concrete results can only be obtained for ultraviolet (UV)-complete scenarios.

In this manuscript, we systematically identify all possible UV-complete models that are predominantly captured, when it comes to LNV phenomena, by $\mathcal{O}_{s}$ at the tree level. All these models are expected to have one thing in common: potentially large contributions to LNV processes combined with insignificant contributions to the light neutrino masses. Such models are expected to manifest themselves most 
efficiently in LNV phenomena like $\mu^{-} \rightarrow e^{+}$-conversion in nuclei, lepton-number-conserving phenomena, including charged-lepton flavor-violating (CLFV) observables, or baryon-number-violating phenomena, including neutronantineutron oscillations. Furthermore, if the rates for $\mu^{-} \rightarrow e^{+}$-conversion in nuclei are indeed close to being accessible, we find that all tree-level realizations of $\mathcal{O}_{s}$ require the existence of new degrees-of-freedom with masses that are within reach of $\mathrm{TeV}$-scale colliders like the LHC.

The following sections are organized as follows. In Sec. II, we study the all-singlets effective operator and illustrate its contributions to neutrino masses, $0 \nu \beta \beta$, and $\mu^{-} \rightarrow e^{+}$-conversion in nuclei. In Sec. III, we list the different UV-complete models that are associated to the all-singlets effective operator at tree level. We discuss various bounds arising from searches for baryon-numberviolating and CLFV processes. In Sec. IV, we comment on some salient collider signatures of the different new particles proposed in this work. Finally, in Sec. VI, we briefly comment on possible extensions of these scenarios which can account for the observed neutrino masses, summarize our results, and conclude.

\section{THE EFFECTIVE ALL-SINGLETS OPERATOR $O_{s}^{\alpha \beta}$}

In the context of particle physics phenomenology, different notations are prevalent in the literature. Before proceeding, we outline the notation used in this paper, which follows that in $[24,25]$. The SM is constructed using only left-chiral Weyl fields: $Q \equiv\left(u_{L}, d_{L}\right), L \equiv\left(\nu_{L}, l_{L}\right)$ are the left-chiral $\mathrm{SU}(2)_{L}$ doublets, while $u^{c}, d^{c}$ and $\ell^{c}$ are the leftchiral $\mathrm{SU}(2)_{L}$ singlet fields. The corresponding Hermitianconjugated fields are identified with a bar (e.g., $\left.\bar{L}, \overline{e^{c}}\right)$. Thus, unbarred fields $L_{\sigma}$ correspond to the $(1 / 2,0)$ representation of the Lorentz algebra, while barred fields $\bar{L}_{\dot{\sigma}} \equiv L_{\sigma}^{\dagger}$ transform under the $(0,1 / 2)$ representation of the algebra. In this terminology, the familiar four-component Dirac spinor consisting of the electron and the positron can be written as $e=\left(e_{L}, \overline{e^{c}}\right)^{T}$. Throughout, color indices are implicit and hence omitted. Also, the SM Higgs doublet is taken to be $H \equiv\left(H^{+}, H^{0}\right)^{T}$, where $H^{0}$ acquires a vacuum expectation value (vev) $v$ to break the $\mathrm{SU}(2)_{L} \times \mathrm{U}(1)_{Y}$ gauge-symmetry spontaneously to $\mathrm{U}(1)_{\mathrm{EM}}$.

Gauge singlets can be formed by either contracting the $\mathrm{SU}(2)_{L}$ indices using the antisymmetric tensor $\epsilon_{i j}$ or the Kronecker $\delta_{i j}$ (for conjugated fields). Additionally, flavor couplings are, unless explicitly shown, implicitly contracted. The flavor structure of the effective operators can be used to infer contributions to different new-physics processes, as we shall see. We also do not explicitly show the Lorentz structure of the different operators. Note that, for the same operator, there can be different contractions associated with the gauge and Lorentz indices.
These different contractions, however, lead to estimates for the rates of the processes of interest which are roughly the same.

An effective operator of mass dimension $d$ is suppressed by $(d-4)$ powers of the effective mass-scale $\Lambda$ of the new physics, i.e.,

$$
\mathcal{L} \supset \frac{g}{\Lambda^{d-4}} \mathcal{O}^{d}+\text { H.c. }
$$

where $g$ s are dimensionless coupling constants. Note that $g$ and $\Lambda$ are not independently defined; one can resolve this issue, e.g., by defining $\Lambda$ such that the largest $g$ is one. The effective scale $\Lambda$ indicates the maximum laboratory energy beyond which the effective-operator description breaks down, i.e., the effective-theory description is valid at energy scales which are at most of order $\Lambda$.

With this arsenal, the dimension-nine all-singlets operators are

$$
\mathcal{O}_{s}^{\alpha \beta}=\ell_{\alpha}^{c} \ell_{\beta}^{c} u^{c} u^{c} \overline{d^{c}} \overline{d^{c}}
$$

where $\ell_{\alpha}^{c} \equiv e^{c}, \mu^{c}$ or $\tau^{c}$. $\mathcal{O}_{s}$ are formed from all the $S U(2)_{L}$-singlet fields. If all quarks are of the same generation, there is only one independent Lorentz contraction: $\left(\ell_{\alpha}^{c}\right)^{\sigma}\left(\ell_{\beta}^{c}\right)_{\sigma}\left(u^{c}\right)^{\rho}\left(u^{c}\right)_{\rho}\left(\overline{d^{c}}\right)_{\dot{\sigma}}\left(\overline{d^{c}}\right)^{\dot{\sigma}}$, where $\sigma, \rho$ and $\dot{\sigma}$ are the Lorentz indices; all other possible contractions are related to this via Fierz transformations. ${ }^{3}$

At different loop-orders, $\mathcal{O}_{s}^{\alpha \beta}$ will contribute to Majorana neutrino masses as well as different LNV processes. In what follows, we estimate in some detail the contributions of these operators to Majorana neutrino masses, $0 \nu \beta \beta$ and $\mu^{-} \rightarrow e^{+}$-conversion in nuclei. The idea [24] is to start with the effective operator, and add SM interactions to generate the relevant processes. The results presented in this section agree with those in [19].

Neutrino Majorana masses are generated by the LNV Weinberg operator [27],

$$
\mathcal{L} \supset \frac{f_{\alpha \beta}}{\Lambda_{W}}\left(L^{\alpha} H\right)\left(L^{\beta} H\right) .
$$

These are dimension-five operators, violate lepton number by two units, and, after electroweak symmetry breaking, lead to neutrino Majorana mass terms, $\mathcal{L} \supset m_{\alpha \beta} \nu^{\alpha} \nu^{\beta}$, $m=f v^{2} / \Lambda_{W} . \Lambda_{W}$ is the effective scale of the Weinberg operator, related to but not the same as $\Lambda$, the effective scale

\footnotetext{
${ }^{3}$ If we consider up-type and down-type quarks of different generations, there are two independent contractions. One can choose those to be $\left(e^{c} \mu^{c}\right)\left(u^{c} t^{c}\right)\left(\overline{d^{c}} \overline{b^{c}}\right)$ and $\left(e^{c} u^{c}\right)\left(\mu^{c} \sigma^{\mu} \overline{d^{c}}\right)\left(t^{c} \sigma_{\mu} \overline{b^{c}}\right)$, where, for convenience, we fix the two different $\ell_{\alpha}^{c}$ to be the electron and the muon and the two different generations of quarks to be the first and third generations. All other contractions can be expressed as combinations of these two.
} 


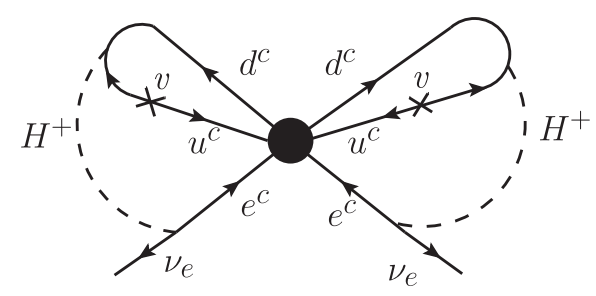

FIG. 1. Four-loop contribution to Majorana neutrino masses from the dimension-nine all-singlets operator $\mathcal{O}_{s}^{e e}$. Given the large loop suppression, the contribution to neutrino masses is only relevant for very low effective scales. The blob represents the effective operator while the $\times$ represents the Higgs-boson vacuum expectation value.

of $\mathcal{O}_{s}^{\alpha \beta}$. Experimental information on neutrino masses point to $\Lambda_{W} \sim 10^{14} \mathrm{GeV}$. Starting from the all-singlets operator, Fig. 1 illustrates how the Weinberg operator is obtained at the four-loop level. In Fig. 1, the blob represents the effective operator $\mathcal{O}_{s}^{e e}$, for concreteness. Clearly, since $\mathcal{O}_{s}^{e e}$ involves only $S U(2)_{L}$-singlet fields, neutrino masses require six Yukawa insertions so one can "reach" the corresponding lepton-doublets $L$ and the Higgs-doublet $H$. The contribution to the neutrino mass matrix can be estimated as

$$
m_{\alpha \beta}=\frac{g_{\alpha \beta}}{\Lambda} \frac{y_{\alpha} y_{\beta}\left(y_{t} y_{b} v\right)^{2}}{\left(16 \pi^{2}\right)^{4}}
$$

where $y$ are the different charged-lepton and quark Yukawa couplings, $\Lambda$ and $g$ are the effective scale and couplings of $\mathcal{O}_{s}^{\alpha \beta}$, respectively, and we assumed third-generation quarks, as these are associated to the largest Yukawa couplings. Note that the $\alpha, \beta$ indices in Eq. (2.4) are not summed over.
Neutrino oscillation data constrain only the neutrino mass-squared differences. Nonetheless, one can use the atmospheric and the solar mass-squared differences to set lower bounds on the masses of the heaviest and the next-toheaviest neutrinos. The atmospheric mass-squared difference, for example, dictates that at least one neutrino has to be heavier than $\sqrt{\left|\Delta m_{32}^{2}\right|} \simeq 0.05 \mathrm{eV}$ [28]. On the other hand, cosmic surveys limit the sum of masses of the neutrinos to be $\lesssim 0.12 \mathrm{eV}$ [29-31]. For concreteness, we assume that the largest element of the neutrino mass matrix lies between $m_{\nu} \in(0.05-0.5) \mathrm{eV}$. In this case, Eq. (2.4) implies that the effective scale of $\mathcal{O}_{s}^{\alpha \beta}[19]$ is

$$
\Lambda \in(100 \mathrm{MeV}-1 \mathrm{GeV}) .
$$

Figure 2 depicts the tree-level, two-loop and four-loop contributions to $0 \nu \beta \beta$ from $\mathcal{O}_{s}^{e e}$. The half-life for such a decay is estimated as [19]

$$
\begin{aligned}
T_{0 \nu \beta \beta}= & \frac{\ln (2)}{\left|g_{e e}\right|^{2}} \frac{\Lambda^{2}}{Q^{11}}\left[\left(\frac{G_{F}}{\sqrt{2}}\right)^{4}\left(\frac{1}{q^{2}}\right)^{2}\left(\frac{y_{t}^{2} y_{b}^{2} y_{e}^{2} v^{2}}{\left(16 \pi^{2}\right)^{4}}\right)^{2}\right. \\
& \left.+\left(\frac{G_{F}}{\sqrt{2}}\right)^{2} \frac{1}{q^{2}}\left(\frac{y_{t} y_{b} y_{e} v}{\left(16 \pi^{2}\right)^{2} \Lambda^{2}}\right)^{2}+\frac{1}{\Lambda^{8}}\right]^{-1} .
\end{aligned}
$$

The effective $Q$-value of the decay process can be extracted from analyses of the data from the KamLAND-Zen experiment [32] and turns out to be $\mathcal{O}(10 \mathrm{MeV})$. The factor of $\left(1 / q^{2}\right)$ comes from the neutrino propagator and is typically of order $100 \mathrm{MeV}$, the inverse distance-scale between nucleons. Combining these, our estimate for the half-life as a function of $\Lambda$ is depicted in the left panel of Fig. 3. For $\mathcal{O}(1)$ couplings, the current lifetime lower-bound $-\Lambda \gtrsim 5 \mathrm{TeV}$-and the
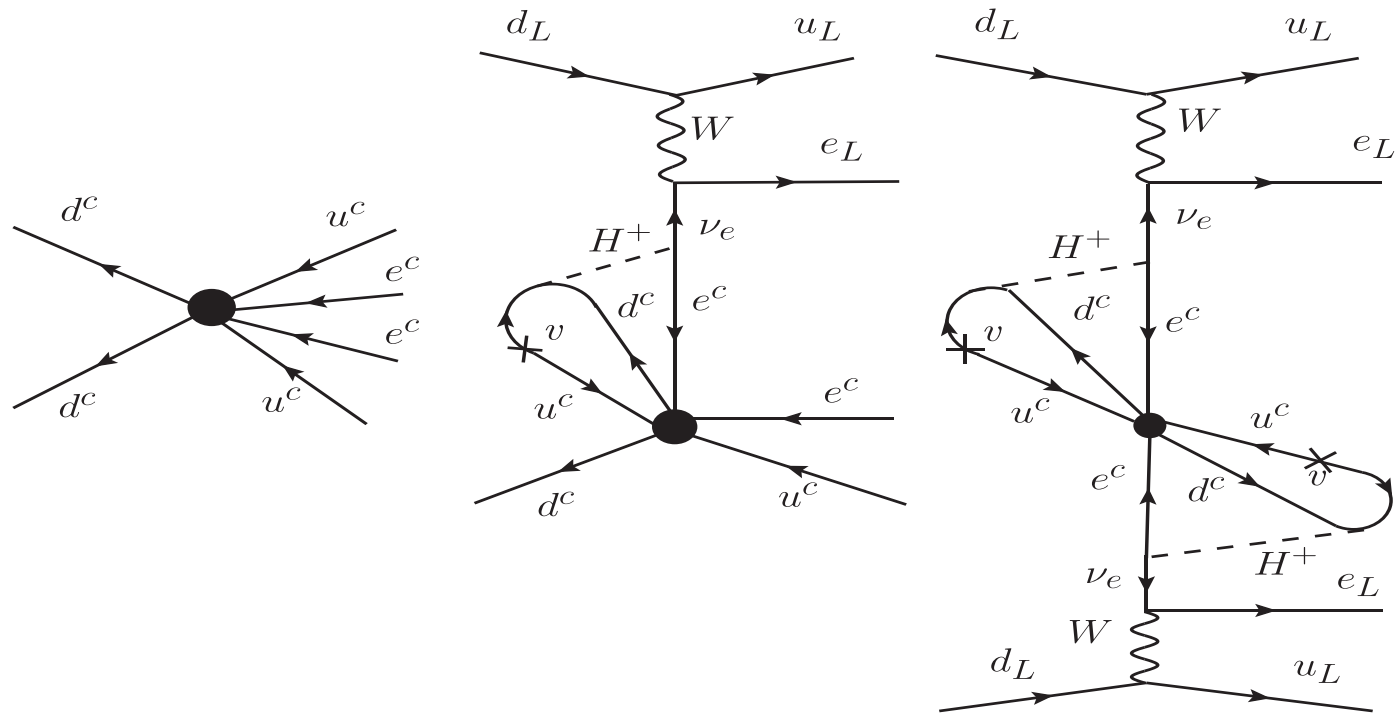

FIG. 2. Feynman diagrams contributing to $0 \nu \beta \beta$ from the dimension-nine all-singlets operator $\mathcal{O}_{s}^{e e}$ at the tree level (left), two-loop level (middle), and four-loop level (right). The blob represents the effective operator while the $\times$ represents the Higgs-boson vacuum expectation value. 

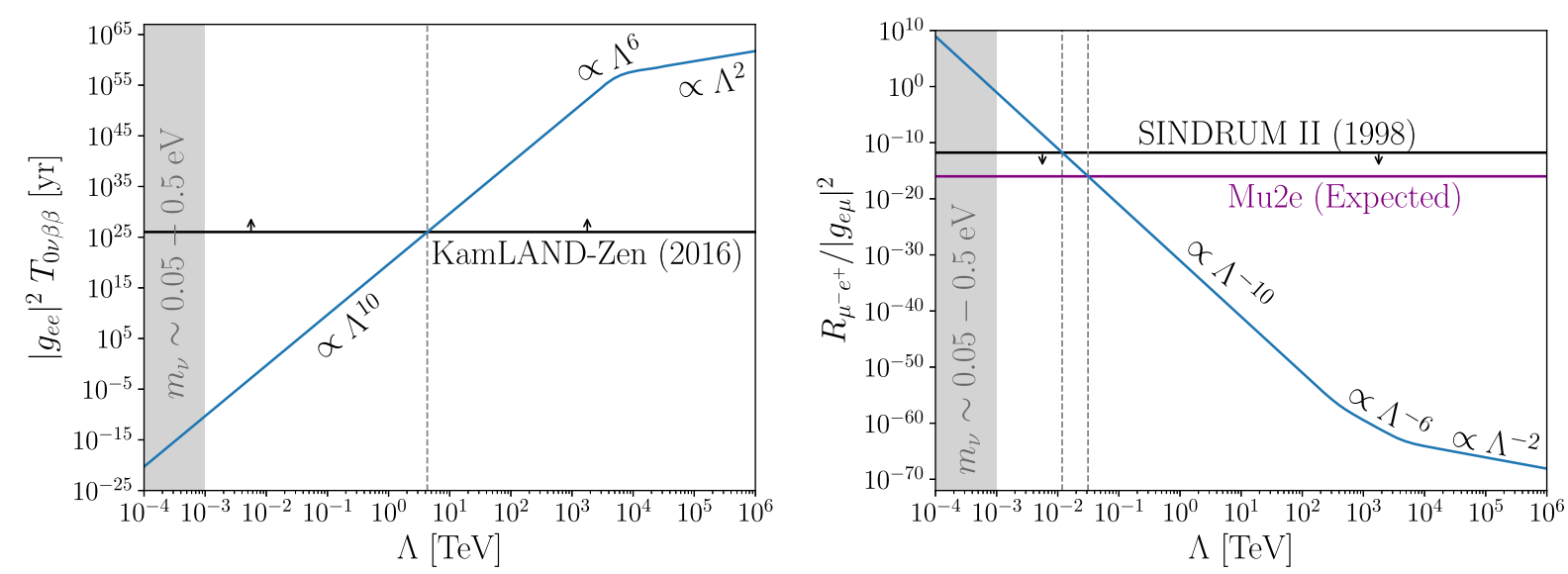

FIG. 3. Left: The lifetime associated to $0 \nu \beta \beta, T_{0 \nu \beta \beta}$, as a function of the cutoff scale $\Lambda$, from the dimension-nine all-singlets operator $\mathcal{O}_{s}^{e e}$. For values $\Lambda \lesssim 10^{4} \mathrm{TeV}$ the lifetime is dominated by the tree-level contribution and scales like $\propto \Lambda^{10}$, whereas for larger values of $\Lambda$, the lifetime is dominated by the four-loop contribution and scales $\propto \Lambda^{2}$. The current experimental bound from KamLAND-Zen is depicted as a horizontal black line. Right: The normalized rate $R_{\mu^{-}} e^{+}$of muon to positron conversion as a function of the cutoff scale $\Lambda$, from the dimension-nine all-singlets operator $\mathcal{O}_{s}^{\mu e}$. For scales $\Lambda \lesssim 10^{2} \mathrm{TeV}$, the tree-level contribution dominates and the rate scales like $\propto \Lambda^{-10}$. For scales $\Lambda \gtrsim 10^{4} \mathrm{TeV}$ the four-loop contribution is most relevant and the rate scales like $\propto \Lambda^{-2}$. Between those regions, the two-loop contribution is most important and the rate scale like $\propto \Lambda^{-6}$. The current experimental bound from SINDRUM II and the sensitivity of $M u 2 e$ are depicted as a horizontal black and purple lines, respectively.

neutrino mass requirements-Eq. (2.5)—are incompatible. This strongly suggests that if there is new physics that manifests itself via $\mathcal{O}_{s}^{e e}$ at the tree level, this new physics is not responsible for generating the observed nonzero neutrino masses.

Figure 4 depicts the tree-level, two-loop and four-loop contributions to $\mu^{-} \rightarrow e^{+}$-conversion from $\mathcal{O}_{s}^{e \mu}$. In order to estimate $R_{\mu^{-}} e^{+}$, as defined in Eq. (1.1), we estimate the muon capture rate, as outlined in [19], to be

$$
\Gamma_{\mu^{-}} \propto\left(\frac{G_{F}}{\sqrt{2}}\right)^{2}\left(\frac{Z_{\mathrm{eff}}^{3}}{\pi\left(a_{0} m_{e} / m_{\mu}\right)^{3}}\right) Q^{2}
$$

where $Z_{\text {eff }}$ is the effective atomic number, $a_{0}$ the Bohr radius, and $Q$ the estimated typical energy of the process, of order the muon mass $m_{\mu}$. While estimating $R_{\mu^{-}} e^{+}$, the term in the second parentheses in Eq. (2.7) cancels out in the ratio, yielding
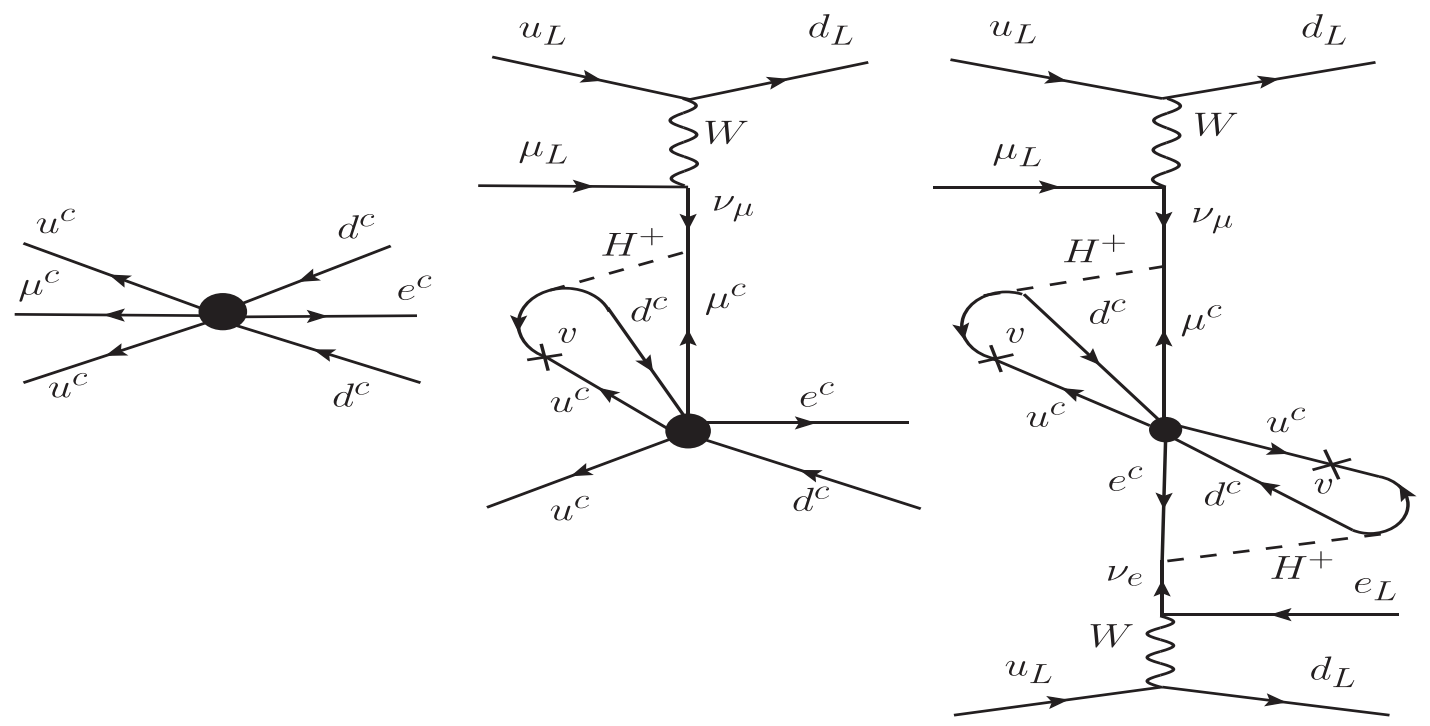

FIG. 4. Feynman diagrams contributing to $\mu^{-} \rightarrow e^{+}$-conversion from the dimension-nine all-singlets operator $\mathcal{O}_{s}^{\mu e}$ at the tree level (left), two-loop level (middle), and four-loop level (right). The blob represents the effective operator while the $\times$ represents the Higgsboson vacuum expectation value. 


$$
\begin{aligned}
R_{\mu^{-} e^{+}}= & \left|g_{e \mu}\right|^{2} \frac{Q^{6}}{\Lambda^{2}}\left[\left(\frac{G_{F}}{\sqrt{2}}\right)^{2}\left(\frac{1}{q^{2}}\right)^{2}\left(\frac{y_{t}^{2} y_{b}^{2} y_{\mu} y_{e} v^{2}}{\left(16 \pi^{2}\right)^{4}}\right)^{2}\right. \\
& \left.+\frac{1}{q^{2}}\left(\frac{y_{t} y_{b} y_{\mu} v}{\left(16 \pi^{2}\right)^{2} \Lambda^{2}}\right)^{2}+\left(\frac{\sqrt{2}}{G_{F}}\right)^{2} \frac{1}{\Lambda^{8}}\right] .
\end{aligned}
$$

The normalized conversion rate for this process as a function of $\Lambda$ is depicted in Fig. 3 along with the current bounds on the process from the SINDRUM II collaboration [33], and the expected Mu2e sensitivity, Eq. (1.2). The current bound from SINDRUM II implies that $\Lambda \gtrsim 10 \mathrm{GeV}$ for $\mathcal{O}(1)$ couplings. Again, the neutrino mass requirements are inconsistent with the existing $\mu^{-} \rightarrow e^{+}$-conversion bounds.

If all $g_{\alpha \beta}$ are of the same magnitude, current constraints on $\Lambda$ from $0 \nu \beta \beta-\Lambda \gtrsim 1 \mathrm{TeV}$ for $g_{e e}$ of order one-would translate into unobservable rates for $\mu^{-} \rightarrow e^{+}$-conversion in nuclei. However, there are no model-independent reasons to directly relate, e.g., $g_{\mu e}$ to $g_{e e}$, hence the bounds from $0 \nu \beta \beta$ need not apply directly to searches for $\mu^{-} \rightarrow e^{+}$conversion. Model-dependent considerations are required in order to explore possible relations between $g_{e e}$ and $g_{\mu e}$. On the other hand, observable rates for $\mu^{-} \rightarrow e^{+}$-conversion require $\Lambda \lesssim 100 \mathrm{GeV}$ and hence new particles with masses around (or below) the weak scale. It is natural to suspect that models associated to such small effective scales are also vulnerable to lepton-number conserving, low-energy observables, especially searches for CLFV. As argued in the introduction, these phenomena can only be addressed within UV-complete models, which we introduce and discuss in the next section.

\section{ULTRAVIOLET COMPLETIONS OF THE EFFECTIVE OPERATOR $O_{s}^{\alpha \beta}$}

Here we discuss tree-level UV-completions of the allsinglets dimension-nine operator $\mathcal{O}_{s}^{\alpha \beta}$, introduced in the previous section, Eq. (2.2). As all fields in the effective operator are fermions, all new interactions involving SM fields are either Yukawa or gauge interactions, i.e., they are all 3-point vertices. Furthermore, relevant interactions involving only new-physics fields are at most also 3-point vertices. This is due to the fact that the operator in question has six fermions in the final state and we are only interested in tree-level realizations of $\mathcal{O}_{s}^{\alpha \beta}$. Since only 3-point vertices are possible, there are only two topologies that lead to $\mathcal{O}_{s}^{\alpha \beta}$ at the tree level $[34,35]$ :

(1) All new particles are bosons. Each boson couples to a pair of SM fermions, and three new-physics bosons define a new interaction vertex. This is depicted in the left panel of Fig. 5. The new-physics bosons can be scalars or vectors.

(2) All new interactions involve one boson and two fermions. The new particles are bosons and fermions and SM fields either couple pair-wise with a

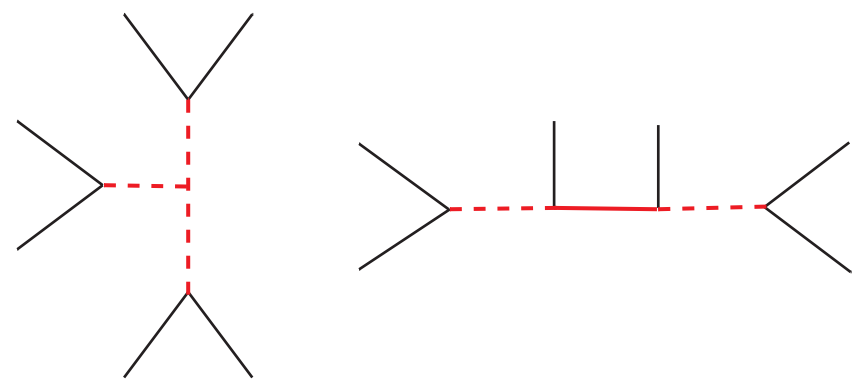

FIG. 5. Topologies that realize the all-singlets dimension-nine operator $\mathcal{O}_{s}$ at tree level. Topology 1 (left) involves only new bosons while Topology 2 (right) requires both new fermions and bosons. In both topologies, the bosons can be scalars or vectors.

new-physics boson or couple to a new-physics boson and a new-physics fermion. This is depicted in the right panel of Fig. 5. Again, the new-physics bosons can be scalars or vectors.

In order to systematically analyze the different internal particles that can appear in Fig. 5, we determine the quantum numbers of pairs and triplets of the external SM fermions of interest. The different combinations of pairs of fermions determine the possible quantum numbers of the bosons in the internal lines in Fig. 5. Similarly, different combinations of triplets of fermions determine the quantum numbers of the potential new fermions in the internal fermion line in Fig. 5 (right).

Table I lists all possible ways of pairing up any two $S U(2)_{L}$-singlet SM fermions. ${ }^{4}$ Generation indices, for both leptons and quarks, have been omitted. Topology 1 can be realized by choosing three bosons with the same quantum numbers as these pairs, keeping in mind that there are two fermions of each type- $u^{c}, d^{c}, \ell^{c}-$ in $\mathcal{O}_{s}^{\alpha \beta}$. For bilinear combinations of the same generation of quarks, only products symmetric in the color indices, i.e., forming a 6 or $\overline{6}$ of $S U(3)_{c}$, exist since, e.g., $\left(u^{c}\right)^{\alpha i}\left(u^{c}\right)_{\alpha}^{j}=$ $-\left(u^{c}\right)_{\alpha}^{i}\left(u^{c}\right)^{\alpha j}=\left(u^{c}\right)^{\alpha j}\left(u^{c}\right)_{\alpha}^{i}$, where $\alpha$ is the dummy Lorentz index and $(i, j)$ are the $S U(3)_{c}$ indices. Here, we will be concentrating on new-physics involving firstgeneration quarks, as we are interested in models that mediate $\mu^{-} \rightarrow e^{+}$-conversion at the tree level (left panel of Fig. 4). Unless otherwise noted, we will not consider models that "mix" different generations of the same quarkflavor.

There are five different "minimal" realizations of Topology 1. Two of them involve heavy scalar bosons only, while the remaining three require new-physics vector and scalar bosons. Of course, one can consider "lessminimal" scenarios where one includes bosons with different quantum numbers associated to the same fermion-pair,

\footnotetext{
${ }^{4}$ Excluding left-handed antineutrinos $\nu^{c}$. We will comment on those later in this section.
} 
TABLE I. Quantum numbers of all possible pairs of the $S U(2)_{L}$ gauge singlet Standard Model fermions $\ell^{c}, u^{c}, \overline{d^{c}}$.

\begin{tabular}{ll}
\hline Pairs (Lorentz) & Representation under $\left(\mathrm{SU}(3)_{\mathrm{C}}, \mathrm{SU}(2)_{\mathrm{L}}\right)_{\mathrm{U}(1)_{\mathrm{Y}}}$ \\
\hline$\ell^{c} \ell^{c}$ (scalar) & $(1,1)_{1} \times(1,1)_{1}=(1,1)_{2}$ \\
$\ell^{c} u^{c}$ (scalar) & $(1,1)_{1} \times(\overline{3}, 1)_{-2 / 3}=(\overline{3}, 1)_{1 / 3}$ \\
$\ell^{c} \overline{d^{c}}$ (vector) & $(1,1)_{1} \times(3,1)_{-1 / 3}=(3,1)_{2 / 3}$ \\
$u^{c} u^{c}$ (scalar) & $(\overline{3}, 1)_{-2 / 3} \times(\overline{3}, 1)_{-2 / 3}=\left(3_{a}, 1\right)_{-4 / 3}+\left(\overline{6}_{s}, 1\right)_{-4 / 3}$ \\
$u^{c} \overline{d^{c}}$ (vector) & $(\overline{3}, 1)_{-2 / 3} \times(3,1)_{-1 / 3}=(1,1)_{-1}+(8,1)_{-1}$ \\
$\overline{d^{c}} \overline{d^{c}}$ (scalar) & $(3,1)_{-1 / 3} \times(3,1)_{-1 / 3}=\left(\overline{3}_{a}, 1\right)_{-2 / 3}+\left(6_{s}, 1\right)_{-2 / 3}$ \\
\hline \hline
\end{tabular}

e.g., the combination $u^{c} \overline{d^{c}}$ can connect to vector bosons in two different $S U(3)_{c}$ representations.

Similarly, Table II lists all possible combinations of three $S U(2)_{L}$-singlet SM fermions. The different new-physics fermions that can make up Topology 2 must have the same quantum numbers as the combinations listed in the table. This list is exhaustive, and to get all possible diagrams, one needs to consider all allowed, distinct permutations of the triplets. In order to realize Topology 2, for each such combination, one needs to consider the possible ways of arranging fermion pairs, listed in Table I. It can be shown that this yields eighteen different "minimal" realizations of Topology 2, not considering the different representations for the same combination of SM fermions.

Next, we want to ensure that, at the tree level, the different new-physics scenarios lead to the all-singlets operator but not to other dimension-nine (or lower dimensional) LNV operators. New particles with the same quantum numbers as some of the combinations in Table I can also couple to pairs of SM fermions that contain the $S U(2)_{L}$-doublets $L, Q$. For example, the pair $\ell^{c} u^{c}$ transforms like a $(\overline{3}, 1)_{1 / 3}$. A scalar that couples to this pair of SM fermions can also couple to $\bar{L} \bar{Q}$, since the latter has identical quantum numbers. These new bosons would lead to, along with the all-singlets operator, other sixfermion operators, including $(\bar{L} \bar{Q})(\bar{L} \bar{Q})\left(\overline{d^{c}} \overline{d^{c}}\right)$ (for a
TABLE III. Pairs of Standard Model fermions that share the same gauge quantum numbers. The pairs of interest here are in bold. The pair $\ell^{c} \ell^{c}$ does not transform like any other pair of SM fields; the same is true of the color-symmetric pairs of $u^{c} u^{c}$ and $d^{c} d^{c}$.

\begin{tabular}{lc}
\hline \hline Fermion pairs transforming as & $\left(\mathrm{SU}(3)_{\mathrm{C}}, \mathrm{SU}(2)_{\mathrm{L}}\right)_{\mathrm{U}(1)_{\mathrm{Y}}}$ \\
\hline$L L, \overline{\ell^{c}} \overline{\nu^{c}}$ & $(1,1)_{-1}$ scalar \\
$\overline{\boldsymbol{d}^{c}} \boldsymbol{u}^{c}, \overline{\ell^{c}} \nu^{c}$ & $(1,1)_{-1}$ vector \\
$\ell^{c} \boldsymbol{u}^{c}, \overline{u^{c}} \overline{d^{c}}, Q^{2}, \bar{L} \bar{Q}, d^{c} \nu^{c}$ & $(\overline{3}, 1)_{1 / 3}$ scalar \\
$\overline{u^{c}} \overline{d^{c}}, Q Q$ & $(6,1)_{1 / 3}$ scalar \\
$\boldsymbol{d}^{c} \boldsymbol{d}^{c}, \overline{u^{c}} \frac{(3,1)_{2 / 3} \text { scalar }}{\bar{\nu}^{c}}$ & $(3,1)_{2 / 3}$ vector \\
$\boldsymbol{d}^{c} \ell^{c}, \bar{L} Q, \overline{u^{c}} \nu^{c}$ & $(3,1)_{-4 / 3}$ scalar \\
$\boldsymbol{u}^{c} \boldsymbol{u}^{c}, \overline{d^{c}} \overline{\ell^{c}}$ & $(1,1)_{0}$ scalar \\
$\nu^{c} \nu^{c}, \overline{\nu^{c}} \overline{\nu^{c}}$ & $(1,1)_{0}$ vector \\
$L \bar{L}, Q \bar{Q}, \ell^{c} \overline{\ell^{c}}, d^{c} \overline{d^{c}}, u^{c} \overline{u^{c}}, \overline{\nu^{c}} \nu^{c}$ & $(8,1)_{0}$ vector \\
$Q \bar{Q}, d^{c} \overline{d^{c}}, u^{c} \overline{u^{c}}$ & \\
\hline \hline
\end{tabular}

complete list, see Tables I, II, and III in [19]). Unlike the all-singlets operator, all other dimension-nine operators saturate the constraints associated to nonzero neutrino masses for $\Lambda$ values that translate into tiny rates for $\mu^{-} \rightarrow e^{+}$-conversion, see Fig. 7 in [19].

In order to systematically address this issue, we list all the relevant SM fermion pairs that transform in the same way in Table III. The pairs relevant for $\mathcal{O}_{s}^{\alpha \beta}$ are shown in bold. From the table, one can see that a new particle that couples to, e.g., $\ell^{c}$ with $u^{c}$ or $\bar{d}^{c}$ can also couple to $\bar{L} \bar{Q}$, and so on. The table reveals that there are two avenues for avoiding unwanted couplings. One is to have one of the new bosons couple to the pair $\ell^{c} \ell^{c}$, which is not degenerate, quantum-number-wise, with any other pair of SM fermions. The other is to add a new fermion and a new boson such that $\ell^{c}$ couples to them in Topology 2. The reason for this is that all other pairings involving $\ell^{c}$ have an unwanted "match," see Table III. This extra requirement drastically reduces the total number of minimal models for the two topologies, and allows us to write down all possible

TABLE II. Quantum numbers of all the possible triplets of $S U(2)_{L}$ gauge singlet Standard Model fermions $\ell^{c}, u^{c}$, $\overline{d^{c}}$ (with at most two identical fields).

\begin{tabular}{lc}
\hline \hline Triplets & Representation under $\left(\mathrm{SU}(3)_{\mathrm{C}}, \mathrm{SU}(2)_{\mathrm{L}}\right)_{\mathrm{U}(1)_{\mathrm{Y}}}$ \\
\hline$\ell^{c} \ell^{c} u^{c}$ & $(1,1)_{1} \times(1,1)_{1} \times(\overline{3}, 1)_{-2 / 3}=(\overline{3}, 1)_{4 / 3}$ \\
$\ell^{c} \ell^{c} \overline{d^{c}}$ & $(1,1)_{1} \times(1,1)_{1} \times(3,1)_{-1 / 3}=(3,1)_{5 / 3}$ \\
$\ell^{c} u^{c} u^{c}$ & $(1,1)_{1} \times(\overline{3}, 1)_{-2 / 3} \times(\overline{3}, 1)_{-2 / 3}=\left(3_{a}, 1\right)_{-1 / 3}+\left(\overline{6}_{s}, 1\right)_{-1 / 3}$ \\
$\ell^{c} \overline{d^{c}} \overline{d^{c}}$ & $(1,1)_{1} \times(3,1)_{-1 / 3} \times(3,1)_{-1 / 3}=\left(\overline{3}_{a}, 1\right)_{1 / 3}+\left(6_{s}, 1\right)_{1 / 3}$ \\
$\ell^{c} u^{c} \overline{d^{c}}$ & $(1,1)_{1} \times(\overline{3}, 1)_{-2 / 3} \times(3,1)_{-1 / 3}=(1,1)_{0}+(8,1)_{0}$ \\
$u^{c} u^{c} \overline{d^{c}}$ & $(\overline{3}, 1)_{-2 / 3} \times(\overline{3}, 1)_{-2 / 3} \times(3,1)_{-1 / 3}=\left[\left(3_{a}, 1\right)_{-4 / 3}+\left(\overline{6}_{s}, 1\right)_{-4 / 3}\right] \times(3,1)_{-1 / 3}$ \\
& $=(\overline{3}, 1)_{-5 / 3}+(6,1)_{-5 / 3}+(\overline{3}, 1)_{-5 / 3}+(1 \overline{5}, 1)_{-5 / 3}$ \\
$u^{c} \overline{d^{c}} \overline{d^{c}}$ & $(\overline{3}, 1)_{-2 / 3} \times(3,1)_{-1 / 3} \times(3,1)_{-1 / 3}=(\overline{3}, 1)_{-2 / 3} \times\left[\left(\overline{3}_{a}, 1\right)_{-2 / 3}+\left(6_{s}, 1\right)_{-2 / 3}\right]$ \\
& $=(3,1)_{-4 / 3}+(\overline{6}, 1)_{-4 / 3}+(3,1)_{-4 / 3}+(15,1)_{-4 / 3}$ \\
\hline \hline
\end{tabular}


TABLE IV. All new particles required for all different tree-level realizations of the all-singlets dimension-nine operator $\mathcal{O}_{s}^{\alpha \beta}$, according to the restrictions discussed in the text. All particles are $S U(2)_{L}$ singlets. The fermions $\psi, \zeta$, and $\chi$ come with a partner ( $\psi^{c}, \zeta^{c}$, and $\chi^{c}$ respectively), not listed. We do not consider fields that would couple to the antisymmetric combination of sameflavor quarks since these cannot couple quarks of the same generation.

\begin{tabular}{lcc}
\hline \hline New particles & $\left(\mathrm{SU}(3)_{\mathrm{C}}, \mathrm{SU}(2)_{\mathrm{L}}\right)_{\mathrm{U}(1)_{\mathrm{Y}}}$ & Spin \\
\hline$\Phi \equiv\left(\overline{l^{c}} \overline{l^{c}}\right)$ & $(1,1)_{-2}$ & Scalar \\
$\Sigma \equiv\left(\overline{u^{c}} \overline{u^{c}}\right)$ & $(6,1)_{4 / 3}$ & Scalar \\
$\Delta \equiv\left(\overline{d^{c}} \overline{d^{c}}\right)$ & $(6,1)_{-2 / 3}$ & Scalar \\
$C \equiv\left(\overline{u^{c}} d^{c}\right)$ & $(1,1)_{1},(8,1)_{1}$ & Vector \\
$\psi \equiv\left(u^{c} l^{c} l^{c}\right)$ & $(\overline{3}, 1)_{4 / 3}$ & Fermion \\
$\zeta \equiv\left(d^{c} \overline{l^{c}} \overline{l^{c}}\right)$ & $(\overline{3}, 1)_{-5 / 3}$ & Fermion \\
$\chi \equiv\left(l^{c} u^{c} u^{c}\right)$ & $(\overline{6}, 1)_{-1 / 3}$ & Fermion \\
$N \equiv\left(l^{c} \overline{d^{c}} u^{c}\right)$ & $(1,1)_{0},(8,1)_{0}$ & Fermion \\
\hline \hline
\end{tabular}

UV completions with no more than three new particles. The final allowed combinations and the corresponding new particles are listed in Table IV. The list is exhaustive, and all possible UV completions of $\mathcal{O}_{s}^{\alpha \beta}$ at the tree level can be implemented with a subset of less than or equal to three of these particles.

It is also important to consider whether new interactions would materialize if neutrino $S U(2)_{L}$-singlet fields, $\nu^{c}$, were also present. Pairings that include $\nu^{c}$ are also included in Table III. Given all constraints discussed above, there are no new couplings involving $\nu^{c}$ other than the neutrino Yukawa coupling and $\nu^{c}$ Majorana masses for new-physics models that do not contain the vector $C^{\mu} \sim(1,1)_{1}$ field. In models that contain $C^{\mu}$, one need also consider the interaction term $\overline{\ell^{c}} \bar{\sigma}^{\mu} \nu_{i}^{c} C_{\mu}$. We return to the left-handed antineutrinos and the mechanism behind neutrino masses in Sec. VI.

In the following subsections we list all the different models. We divide them into different categories. Some models contain new vector bosons, others contain only new-physics scalars or fermions. Since all new particles need to be heavy, including potential new vector bosons, no-vectors models are easier to analyze since, as is wellknown, consistent quantum field theories with massive vector bosons require extra care. There are, altogether, eight models: four with and four without new massive vector fields. We discuss the no-vectors models first. We will also broadly distinguish models based on whether they also lead to the violation of baryon-number conservation and whether any flavor-structure naturally arises.

\section{A. No-vectors models}

Here, all no-vectors models are discussed in turn. Models are named according to the new-physics field content, see
Table IV. Explicitly, they are (1) $\zeta \Phi \Sigma$, (2) $\chi \Delta \Sigma$, (3) $\psi \Delta \Phi$, and (4) $\Phi \Sigma \Delta$. The first three realize $\mathcal{O}_{s}^{\alpha \beta}$ via topology 2 [Fig. 5(right)] while the last one realizes $\mathcal{O}_{s}^{\alpha \beta}$ via topology 1 [Fig. 5(left)].

\section{Model $\zeta \Phi \mathbf{\Sigma}$}

Here, the SM particle content is augmented by a couple of vectorlike fermions $\zeta \equiv(\overline{3}, 1)_{-5 / 3}$ and $\zeta^{c} \equiv(3,1)_{5 / 3}$, the color-singlet scalar $\Phi \sim(1,1)_{-2}$, and the colored scalar $\Sigma \sim(6,1)_{4 / 3}$. The most general renormalizable Lagrangian is

$$
\begin{aligned}
\mathcal{L}_{\zeta \Phi \Sigma}= & \mathcal{L}_{\mathrm{SM}}+\mathcal{L}_{\mathrm{kin}}+y_{\Phi \alpha \beta} \Phi \ell_{\alpha}^{c} \ell_{\beta}^{c}+y_{\Sigma u} \Sigma u^{c} u^{c} \\
& +y_{\Phi \zeta^{c}} \Phi \zeta^{c} d^{c}+y_{\Sigma \zeta} \Sigma \zeta d^{c}+m_{\zeta} \zeta \zeta^{c} \\
& +V(\Phi, \Sigma, 0)+\text { H.c. }
\end{aligned}
$$

where $\mathcal{L}_{\mathrm{SM}}$ is the SM Lagrangian, $\mathcal{L}_{\text {kin }}$ contains the kineticenergy terms for the new particles, and $V(\Phi, \Sigma, 0)$ is the most general scalar potential involving the scalars $\Phi, \Sigma$, written out explicitly in Appendix A. By design, lepton number is violated by two units but it is conserved in the limit where any of the new Yukawa couplings vanishes. On the other hand, baryon number is conserved. In units where the quarks have baryon-number one, $\Sigma$ can be assigned baryon-number $+2, \zeta, \zeta^{c}$ baryon-number $-1,+1$, respectively, and $\Phi$ baryon-number zero.

It is easy to check that this model realizes $\mathcal{O}_{s}^{\alpha \beta}$ via topology 2 [Fig. 5(right)] and

$$
\frac{g_{\alpha \beta}}{\Lambda^{5}} \equiv \frac{y_{\Phi \alpha \beta} y_{\Phi \zeta}^{*} y_{\Sigma \zeta}^{*} y_{\Sigma u}}{M_{\Phi}^{2} M_{\Sigma}^{2} m_{\zeta}} .
$$

Here, $y_{\Phi \alpha \beta}$ controls the lepton-flavor structure of the model. $\mu^{-} \rightarrow e^{+}$-conversion rates are proportional to $\left|y_{\Phi e \mu}\right|^{2}$, while those for $0 \nu \beta \beta$ are proportional to $\left|y_{\Phi e e}\right|^{2}$.

The new-physics states will also mediate CLFV phenomena, sometimes at the tree level. In what follows, we write down the effective operators that give rise to different CLFV processes, and estimate bounds on the effective scales of these operators. The CLFV observables of interest are:

(1) $\mu^{ \pm} \rightarrow e^{ \pm} e^{ \pm} e^{\mp}$ decay: The effective Lagrangian giving rise to this decay, generated at the tree level, is

$$
\mathcal{L}_{\mu \rightarrow 3 e}=\frac{y_{\Phi e \mu} y_{\Phi e e}^{*}}{M_{\Phi}^{2}}\left(\mu^{c} e^{c}\right)\left(\overline{e^{c}} \overline{e^{c}}\right),
$$

and the relevant Feynman diagram is depicted in the left panel of Fig. 6. The strongest bounds on $\mu^{+} \rightarrow e^{+} e^{-} e^{+}$come from the SINDRUM spectrometer experiment [36]: 

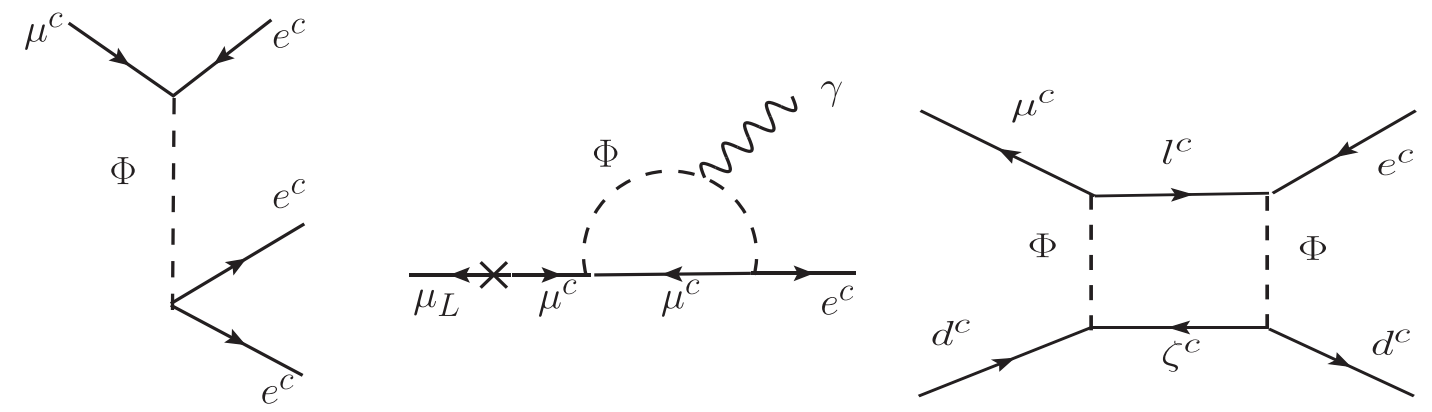

FIG. 6. Feynman diagrams contributing to the CLFV processes $\mu \rightarrow 3 e$ (left), $\mu^{+} \rightarrow e^{+} \gamma$ (middle), and $\mu^{-} \rightarrow e^{-}$-conversion (right) in model $\zeta \Phi \Sigma$.

$$
\operatorname{Br}\left(\mu^{+} \rightarrow e^{+} e^{-} e^{+}\right)<1.0 \times 10^{-12} .
$$

Assuming the phase-space distributions are similar to those of ordinary $\mu$-decay $\left(\mu \rightarrow e \bar{\nu}_{e} \nu_{\mu}\right)$, this translates into $[37,38]$

$$
\frac{\left|y_{\Phi e \mu} y_{\Phi e e}^{*}\right|^{2}}{M_{\Phi}^{4}} \leq 1.4 \times 10^{-22} \mathrm{GeV}^{-4}
$$

or $M_{\Phi} \geq 290 \mathrm{TeV}$ for $\mathcal{O}(1)$ couplings. The Mu3e experiment, under construction at PSI, aims to reach sensitivities better than $10^{-15}$ on this channel [39] and hence sensitivity to $\Phi$-masses around $1000 \mathrm{TeV}$ [7].

(2) $\mu^{+} \rightarrow e^{+} \gamma$ decay: At the one-loop level, $\Phi$-exchange also mediates, as depicted in the middle panel of Fig. $6, \mu^{+} \rightarrow e^{+} \gamma$. The effective operator governing $\mu \rightarrow e \gamma$ is

$$
\mathcal{L}_{\mu^{+} \rightarrow e^{+} \gamma}=\frac{y_{\Phi \mu \mu}^{*} y_{\Phi \mu e}(2 e) y_{\mu}}{16 \pi^{2} M_{\Phi}^{2}}(L \bar{H}) \sigma^{\alpha \beta} e^{c} F_{\alpha \beta},
$$

where $y_{\mu}$ is the muon Yukawa coupling. Experimentally, the most stringent constraints come from the MEG experiment at PSI [40]

$$
\operatorname{Br}\left(\mu^{+} \rightarrow e^{+} \gamma\right)=4.2 \times 10^{-13} .
$$

Using results from [41], we get

$$
\begin{aligned}
\operatorname{Br}\left(\mu^{+} \rightarrow e^{+} \gamma\right) & \approx 5.3 \times 10^{-6} \frac{\left|y_{\Phi \mu \mu}^{*} y_{\Phi \mu e}\right|^{2}}{M_{\Phi}^{4}(\mathrm{TeV})} \\
& <4.2 \times 10^{-13},
\end{aligned}
$$

which leads to $M_{\Phi} \gtrsim 60 \mathrm{TeV}$, given $\mathcal{O}(1)$ couplings. As expected, the $\mu^{+} \rightarrow e^{+} \gamma$ bound is weaker than that of $\mu \rightarrow 3 e$ as the former is loop-suppressed. The upgraded MEG-II experiment plans to reach a sensitivity of $10^{-14}$ with three years of data taking [42].

(3) $\mu^{-} \rightarrow e^{-}$-conversion in nuclei: In this model, $\mu^{-} \rightarrow e^{-}$-conversion occurs at the one-loop level, as depicted in the right panel of Fig. 6. The effective Lagrangian can be estimated as

$$
\mathcal{L}_{\mu \rightarrow e}=\frac{y_{\Phi \mu \beta}^{*} y_{\Phi e \beta} y_{\Phi \zeta^{c}} y_{\Phi \zeta^{c}}^{*}}{16 \pi^{2} \Lambda^{2}}\left(\overline{\mu^{c}} e^{c}\right)\left(\overline{d^{c}} d^{c}\right)
$$

where $\Lambda$ is the effective scale, a function of $M_{\Phi}$ and $M_{\zeta}$, and the lepton-index $\beta$ is summed over. Note that this operator is also sensitive to the $y_{\Phi e \tau}$ and $y_{\Phi \mu \tau}$ couplings. An extra contribution comes from the middle panel of Fig. 6, where the photon is put offshell, and radiates a $q \bar{q}$ pair.

The SINDRUM II experiment at PSI constrains $\mu^{-} \rightarrow e^{-}$-conversion in gold [33]:

$$
\begin{aligned}
R_{\mu^{-} e^{-}}^{\mathrm{Au}} & \equiv \frac{\Gamma\left(\mu^{-}+\mathrm{Au} \rightarrow e^{-}+\mathrm{Au}\right)}{\Gamma\left(\mu^{-}+\mathrm{Au} \rightarrow \nu_{\mu}+\mathrm{Pt}\right)} \\
& <7 \times 10^{-13}(90 \% \mathrm{CL}) .
\end{aligned}
$$

Using Eq. (2.7), we estimate [37]

$$
R_{\mu^{-} e^{-}}=\left|\frac{\sqrt{2}}{G_{F}} \frac{y_{\Phi \mu \beta}^{*} y_{\Phi e \beta} y_{\Phi \zeta^{c}} y_{\Phi \zeta^{c}}^{*}}{16 \pi^{2} \Lambda^{2}}\right|^{2}
$$

For $\mathcal{O}(1)$ couplings, this yields $\Lambda \geq 30 \mathrm{TeV}$. Stronger sensitivity is expected from the next-generation experiments COMET [15], DeeMe [16], and $M u 2 e$ [17], as discussed in the introduction. Ultimately, one would be sensitive to $\Lambda$ scales up to a few $100 \mathrm{TeV}$.

(4) Muonium-antimuonium oscillations $\left(\mu^{+} e^{-} \rightarrow \mu^{-} e^{+}\right)$: Muonium $(\mathrm{Mu})$ is the bound state of an $e^{-}$and a $\mu^{+}$, whereas its anti-partner, the antimuonium $(\overline{\mathrm{Mu}})$ is the bound state of an $e^{+}$and a $\mu^{-}$. Muoniumantimuonium oscillation is a process where muonium converts to antimuonium, thereby changing both electron-number and muon-number by two units [37]. Here, the effective Lagrangian governing this process at the tree level is 


$$
\mathcal{L}_{\mathrm{Mu}-\overline{\mathrm{Mu}}}=\frac{y_{\Phi \mu \mu} y_{\Phi e e}^{*}}{M_{\Phi}^{2}}\left(\mu^{c} \mu^{c}\right)\left(\overline{e^{c}} \overline{e^{c}}\right)
$$

The probability that a $\mathrm{Mu}$ bound state at $t=0$ is detected as a $\overline{\mathrm{Mu}}$ bound state at a later time is proportional to $\left(y_{\Phi \mu \mu} y_{\Phi e e}^{*}\right) / M_{\Phi}^{2}$. The upper limit quoted by the PSI experiment [43] yields $\left(y_{\Phi \mu \mu} y_{\Phi e e}^{*}\right) / M_{\Phi}^{2} \lesssim 0.002 G_{F}$ or

$$
\frac{\left|y_{\Phi \mu \mu} y_{\Phi e e}^{*}\right|}{M_{\Phi}^{2}} \leq 2.5 \times 10^{-8} \mathrm{GeV}^{-2},
$$

which implies $M_{\Phi} \geq 6.3 \mathrm{TeV}$ for $\mathcal{O}(1)$ couplings.

(5) Lepton-lepton scattering: $\Phi$-exchange will also mediate intermediate and high-energy scattering processes including $e^{ \pm} \mu^{ \pm} \rightarrow e^{ \pm} \mu^{ \pm}, e^{+} e^{-} \rightarrow e^{+} e^{-}$, and $e^{+} e^{-} \rightarrow \mu^{+} \mu^{-}$. If $M_{\Phi}$ is much larger than the center-of-mass-energies of interest, the following tree-level effective Lagrangian applies:

$$
\mathcal{L}_{e \mu}=\frac{y_{\Phi e \mu} y_{\Phi e \mu}^{*}}{M_{\Phi}^{2}} e^{c} \mu^{c} \bar{e}^{c} \bar{\mu}^{c}
$$

Measurements of $\sigma\left(e^{+} e^{-} \rightarrow \mu^{+} \mu^{-}\right)$and $\sigma\left(e^{+} e^{-} \rightarrow e^{+} e^{-}\right)$at LEP [44] can be translated into constraints on the effective scale of the operator above,

$\frac{y_{\Phi e \mu} y_{\Phi e \mu}^{*}}{2 M_{\Phi}^{2}} \leq \frac{4 \pi}{\Lambda_{\mu}^{2}} \quad$ and $\quad \frac{y_{\Phi e e} y_{\Phi e e}^{*}}{2 M_{\Phi}^{2}} \leq \frac{4 \pi}{2 \Lambda_{e}^{2}}$,

where $\Lambda_{\mu} \approx 9.3 \mathrm{TeV}$ and $\Lambda_{e} \approx 8.9 \mathrm{TeV}$. These translate into $M_{\Phi} \gtrsim 2.5 \mathrm{TeV}$, given $\mathcal{O}(1)$ couplings.

(6) Anomalous magnetic moments: There is a wellknown discrepancy between the experimental value [45] and the SM prediction [46,47] of the anomalous magnetic moment of the muon, $10.1 \times 10^{-10}<$ $a_{\mu}^{\exp }-a_{\mu}^{\mathrm{SM}}<42.1 \times 10^{-10}$ at the $2 \sigma$ level. The doubly charged $\Phi$-scalar will contribute to the muon $(g-2)$ at the one-loop level. The corresponding Feynman diagrams are quite similar to the middle panel of Fig. 6 with the external electron replaced by a muon. In the limit $\Phi$ is much heavier than muons and electrons, the resulting contribution is [48] (see also $[49,50])$

$$
\Delta a_{\mu}=-\frac{m_{\mu}^{2}\left(y_{\Phi e \mu} y_{\Phi e \mu}^{*}+y_{\Phi \mu \mu} y_{\Phi \mu \mu}^{*}\right)}{6 \pi^{2} M_{\Phi}^{2}} .
$$

The negative sign of the contribution indicates that this type of new physics will not help alleviate the discrepancy. We can, nonetheless, derive a limit from the $g-2$ measurement by requiring the absolute value of the contribution to be less than the discrepancy, which leads to $M_{\Phi} \gtrsim 734 \mathrm{GeV}$, given the $\mathcal{O}(1)$ couplings. This bound is weaker than most of the previous ones discussed here. The Muon $g-2$ experiment, currently taking data at Fermilab, is ultimately expected to improve on the uncertainty of the muon $g-2$ by roughly a factor of two [51].

A subset of the bounds estimated here is summarized in Fig. 11. Not surprisingly, if all couplings of interest are of order one, constraints from $\mu \rightarrow 3 e$ are the strongest and translate into $M_{\Phi}$ values that exceed hundreds of TeV. CLFV observables do not constrain, directly, $m_{\zeta}$ or $M_{\Sigma}$, while searches for $\mu^{-} \rightarrow e^{-}$-conversion are sensitive to both $M_{\Phi}$ and $m_{\zeta}$. Since both $\zeta$ and $\Sigma$ are colored, we expect LHC searches for exotic fermions or scalars to constrain, conservatively, $m_{\zeta}, M_{\Sigma} \gtrsim 500 \mathrm{GeV}$. We return to this issue briefly in Sec. IV. Putting it all together, if all new-physics couplings are of order one, searches for CLFV imply upper bounds on the rate for $\mu^{-} \rightarrow e^{+}$-conversion that are much stronger than the sensitivity of next-generation experiments.

Most of the CLFV bounds can be avoided, along with those from $0 \nu \beta \beta$, if the flavor-structure of the new physics is not generic. In particular, in the limit where $y_{\Phi e \mu}$ is much larger than all other $y_{\Phi \alpha \beta}$ couplings, most of the constraints above become much weaker. This can be understood by noting that $\mu^{-} \rightarrow e^{+}$-conversion preserves an $L_{\mu}-L_{e}$ (muon-number minus electron-number) global symmetry while the physics processes $\mu \rightarrow 3 e, \mu \rightarrow e \gamma, \mu^{ \pm} \rightarrow e^{ \pm}$conversion, and $0 \nu \beta \beta$ all violate $L_{\mu}-L_{e}$ by two units, while $\mathrm{Mu}-\overline{\mathrm{Mu}}$-oscillations violate $L_{\mu}-L_{e}$ by four units. In other words, if only the $\Phi \mu^{c} e^{c}$-coupling $y_{\Phi \mu e}$ is nonzero, the new-physics portion of the Lagrangian respects an $L_{\mu}-L_{e}$ global symmetry and all CLFV bounds vanish to a very good approximation. The flavor-diagonal constraints from LEP and the muon $g-2 \mathrm{do}$, however, apply, but are of order $1 \mathrm{TeV}$ for $y_{\Phi \mu e}$ of order one, much less severe. This is a property of all new-physics scenarios that contain the $\Phi$-field since, in these scenarios, the only coupling of the leptons to the new degrees-of-freedom is the one to $\Phi$.

\section{Model $\chi \Delta \Sigma$}

Here, the SM particle content is augmented by a couple of vectorlike fermions $\chi \sim(\overline{6}, 1)_{-1 / 3}$ and $\chi^{c} \sim(6,1)_{1 / 3}$, and two colored scalars $\Sigma \sim(6,1)_{4 / 3}$ and $\Delta \sim(6,1)_{-2 / 3}$. The most general renormalizable Lagrangian is

$$
\begin{aligned}
\mathcal{L}_{\chi \Delta \Sigma}= & \mathcal{L}_{\mathrm{SM}}+\mathcal{L}_{\mathrm{kin}}+y_{\Sigma u} \Sigma u^{c} u^{c}+y_{\Delta d} \Delta d^{c} d^{c}+y_{\Delta \chi} \Delta \bar{\chi} \bar{\chi} \\
& +y_{\Delta \chi^{c}} \Delta \chi^{c} \chi^{c}+y_{\Sigma \alpha} \bar{\Sigma} \chi^{c} \ell_{\alpha}^{c}+y_{\Delta \alpha} \Delta \chi \ell_{\alpha}^{c} \\
& +m_{\chi} \chi \chi^{c}+V(0, \Sigma, \Delta)+\text { H.c. }
\end{aligned}
$$

where $\mathcal{L}_{\mathrm{SM}}$ is the SM Lagrangian, $\mathcal{L}_{\text {kin }}$ contains the kineticenergy terms for the new particles, and $V(0, \Sigma, \Delta)$ is the most general scalar potential involving the scalars $\Delta, \Sigma$, 
written out explicitly in Appendix A. The operator $\mathcal{O}_{s}^{\alpha \beta}$ is realized at the tree level with topology 2 , and the effective scale is given by

$$
\frac{g_{\alpha \beta}}{\Lambda^{5}} \equiv \frac{y_{\Sigma u} y_{\Sigma \alpha} y_{\Delta \beta} y_{\Delta d}^{*}}{M_{\Sigma}^{2} M_{\Delta}^{2} m_{\chi}}
$$

The $\mu^{-} \rightarrow e^{+}$-conversion rates are proportional to $\left|y_{\Sigma e} y_{\Delta \mu}+y_{\Sigma \mu} y_{\Delta e}\right|^{2}$, while those for $0 \nu \beta \beta$ are proportional to $\left|y_{\Sigma e} y_{\Delta e}\right|^{2}$.

Like the previous example, this model also allows for a rich set of CLFV processes. The CLFV observables of interest are:

(1) $\mu^{+} \rightarrow e^{+} \gamma$ decay: This is generated at the one-loop level, as depicted in the left panel of Fig. 7. There is a similar diagram with $\Delta$ and $\chi^{c}$ in the loop. The effective Lagrangian for this process is

$$
\mathcal{L}_{\mu^{+} \rightarrow e^{+} \gamma}=\frac{y_{\Delta \mu} y_{\Delta e}^{*}(2 e) y_{\mu}}{16 \pi^{2} \Lambda^{2}}(L \bar{H}) \sigma^{\alpha \beta} e^{c} F_{\alpha \beta},
$$

where $\Lambda$ is a function of $M_{\Delta}$ and $m_{\chi}$. The bounds for this model are similar to the ones calculated in Eq. (3.8).

(2) $\mu^{ \pm} \rightarrow e^{ \pm} e^{ \pm} e^{\mp}$ decay: Unlike the previous model, here $\mu \rightarrow 3 e$ only occurs at the one-loop level. One contribution is obtained from the diagram in the left panel of Fig. 7, where the photon is off-shell and can "decay" into an $e^{+} e^{-}$pair. As far as this contribution is concerned, the rate for $\mu \rightarrow 3 e$ is suppressed relative to that for the $\mu \rightarrow e \gamma$ decay. There are also box-diagrams, including the one depicted in the right panel of Fig. 7, which could also contribute significantly. Figure 7(right) gives rise to the effective Lagrangian

$$
\mathcal{L}_{\mu \rightarrow 3 e}=\frac{y_{\Delta \mu} y_{\Delta e} y_{\Delta e}^{*} y_{\Delta e}^{*}}{16 \pi^{2} \Lambda^{2}}\left(\overline{\mu^{c}} e^{c}\right)\left(\overline{e^{c}} e^{c}\right)
$$

where $\Lambda$ is a function of $M_{\Delta}$ and $m_{\chi}$. Using Eq. (3.4), current data constrain $\Lambda \geq 23 \mathrm{TeV}$ assuming order one couplings. A similar box-diagram exists with $\chi^{c}$ and $\Sigma$ in the loop; its contribution turn out to be of the same order.
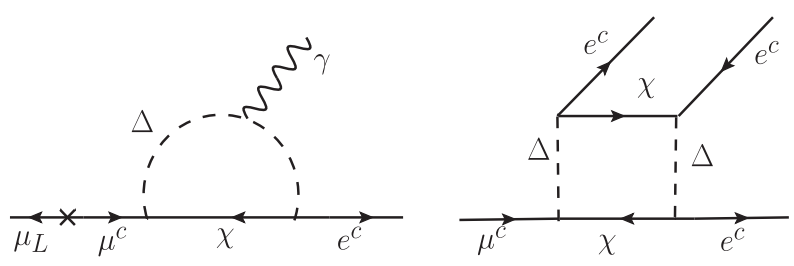

FIG. 7. Feynman diagrams for the CLFV processes $\mu \rightarrow e \gamma$ (left) and $\mu \rightarrow 3 e$ [box-diagram] (right), in model $\chi \Delta \Sigma$.
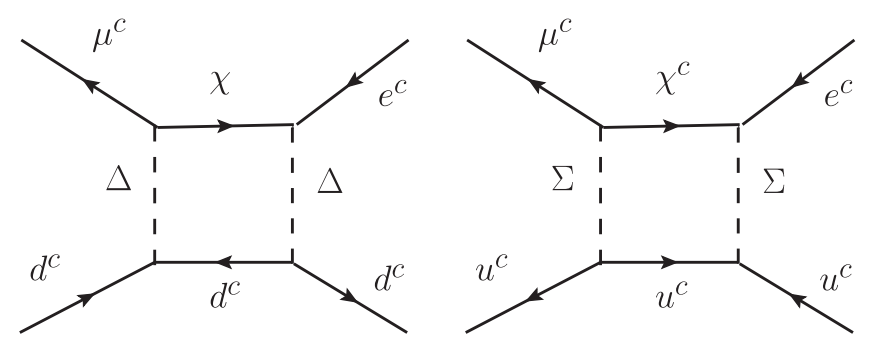

FIG. 8. Feynman diagrams (box-diagrams) contributing to the CLFV process $\mu^{-} \rightarrow e^{-}$-conversion, in model $\chi \Delta \Sigma$.

(3) $\mu^{-} \rightarrow e^{-}$-conversion in nuclei: In this model, $\mu^{-} \rightarrow e^{-}$-conversion also occurs at the one-loop level, as depicted in Fig. 8. The effective Lagrangian can be estimated as

$$
\begin{aligned}
\mathcal{L}_{\mu \rightarrow e}= & \left(\frac{y_{\Delta \mu}^{*} y_{\Delta d} y_{\Delta d}^{*} y_{\Delta e}}{16 \pi^{2} \Lambda_{\Delta \chi}^{2}}+\frac{y_{\Sigma \mu}^{*} y_{\Sigma u}^{*} y_{\Sigma u} y_{\Sigma e}}{16 \pi^{2} \Lambda_{\Sigma \chi}^{2}}\right) \\
& \times\left(\overline{\mu^{c}} e^{c}\right)\left(\overline{d^{c}} d^{c}\right)
\end{aligned}
$$

where the subscripts on $\Lambda$ denotes the dependence on the masses of the new particles. As in the previous model, this process can also proceed through the transition-magnetic-moment channel, where the photon emits a quark-antiquark pair. The bounds arising on the effective scale for $\mathcal{O}(1)$ couplings are similar to ones obtained in the previous model (model $\zeta \Phi \Sigma)$. There exists a dimensionten operator $\left(d^{c} d^{c} \overline{d^{c}} \overline{d^{c}} \ell^{c}[\sigma \cdot \partial] \overline{\ell^{c}}\right)$, which can be dressed as the process $n \mu^{-} \rightarrow n e^{-}$. The relevant amplitude is

$$
\mathcal{A}=\frac{y_{\Delta d} y_{\Delta d}^{*} y_{e \Delta} y_{\mu \Delta}^{*}}{M_{\Delta}^{4} m_{\chi}^{2}}\left(d^{c} d^{c} \overline{d^{c}} \overline{d^{c}} \ell^{c}[p \cdot \sigma] \overline{\ell^{c}}\right)
$$

where $p$ is the typical four-momentum associated to the process. Note that there is an analogous contribution to $p \mu^{-} \rightarrow p e^{-}$. This will also mediate $\mu^{-} \rightarrow e^{-}$-conversion in nuclei. However, this is an effective operator of very high energy-dimension and hence suppressed.

(4) Muonium-antimuonium oscillations and lepton scattering: Unlike the previous model (model $\zeta \Phi \Sigma$ ), this model does not allow for tree-level muoniumantimuonium oscillation, or lepton-lepton scattering. One can, of course, have these processes at the one-loop level through diagrams like the right panel of Fig. 7. The bounds arising from these processes are not expected to be competitive with the other leptonic bounds.

(5) Anomalous magnetic moments: there is a newphysics contribution to the anomalous magnetic moment of the muon and the electron at one-loop 
(e.g., a $\Delta, \chi$ loop). The situation here is very similar to the one discussed in model $\zeta \Phi \Sigma$.

A subset of the bounds estimated here are summarized in Fig. 11. As in the previous model, in the absence of flavorstructure in the new-physics sector, CLFV constraints, along with those from $0 \nu \beta \beta$-searches, overwhelm the sensitivity of future searches for $\mu^{-} \rightarrow e^{+}$-conversion. In this model, it is also possible to consistently assign $L_{\mu}-L_{e}$ charges to the heavy fields and therefore eliminate the processes listed above. For example, if we assign charge +1 to $\chi$ and charge -1 to $\chi^{c}$, only $\mu^{c}$ couples to $\chi$ and only $e^{c}$ couples to $\chi^{c}$. This can automatically prevent the above processes from taking place with a sizable rate. Note that this charge assignment will render some of the other newphysics couplings zero, e.g., $y_{\Delta \chi}$ and $y_{\Delta \chi^{c}}$.

Unlike model $\zeta \Phi \Sigma$, here baryon number is explicitly violated. We note that the Lagrangian Eq. (3.17) has an accidental $Z_{2}$ symmetry under which all lepton-fields, along with $\chi$ and $\chi^{c}$, are odd. This implies that nucleon decays into leptons are not allowed (e.g., $p \rightarrow \pi^{0}+e^{+}$or $n \rightarrow \pi^{0}+\nu$ ) and, for example, the proton is stable. There are, nonetheless, a few relevant baryon-number-violating (BNV) constraints:

(1) Neutron-antineutron $(n-\bar{n})$ oscillations: at the tree level, the model mediates neutron-antineutron oscillations, which violate baryon number by two units, as depicted in Fig. 9. The effective Lagrangian for such a process is the dimension-nine operator

$$
\mathcal{L}_{n-\bar{n}}=\frac{y_{\Sigma u} y_{\Delta d}^{2} m_{\Sigma \Delta}}{M_{\Delta}^{4} M_{\Sigma}^{2}}\left(u^{c} d^{c} d^{c}\right)^{2}
$$

Here $m_{\Sigma \Delta}$ is a parameter in the scalar potential, see Appendix A. The Institut Laue-Langevin (ILL) experiment at Grenoble yields the best bounds on free $n-\bar{n}$ oscillations using neutrons from a reactor source [52]. The bounds are typically quoted on the transition matrix element of the effective Hamiltonian, $\delta m=\left\langle\bar{n}\left|H_{\text {eff }}\right| n\right\rangle$ and are

$$
\tau_{n-\bar{n}} \equiv \frac{1}{|\delta m|} \gtrsim 10^{8} \mathrm{sec}
$$

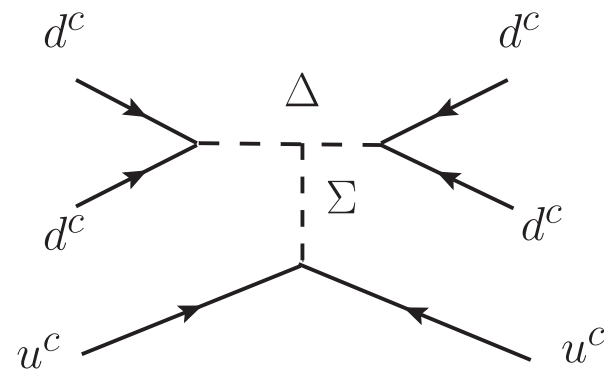

FIG. 9. Tree-level Feynman diagram that mediates $n-\bar{n}$ oscillations in model $\chi \Delta \Sigma$.
Using Eq. (3.23) [53],

$$
\begin{aligned}
\left\langle\bar{n}\left|H_{\text {eff }}\right| n\right\rangle & =\frac{y_{\Sigma u} y_{\Delta d}^{2} m_{\Sigma \Delta}}{M_{\Delta}^{4} M_{\Sigma}^{2}}\left\langle\bar{n}\left|\left(u^{c} d^{c} d^{c}\right)^{2}\right| n\right\rangle \\
& =\frac{y_{\Sigma u} y_{\Delta d}^{2} m_{\Sigma \Delta}}{M_{\Delta}^{4} M_{\Sigma}^{2}} \Lambda_{\mathrm{QCD}}^{6},
\end{aligned}
$$

where we estimate the nucleon matrix-element to be of order $\Lambda_{\mathrm{QCD}}$. Assuming $\mathcal{O}(1)$ couplings, $\Lambda_{\mathrm{QCD}}=180 \mathrm{MeV}$, and $m_{\Sigma \Delta} \sim M_{\Sigma} \sim M_{\Delta} \sim \Lambda$, this translates into

$$
\Lambda \gtrsim 350 \mathrm{TeV} .
$$

(2) BNV processes with LNV: The model also allows for $\mathrm{BNV}$ processes that violate lepton number related to the effective dimension-twelve operator $\left(d^{c} d^{c} d^{c} \bar{\ell}^{c}\right)^{2}$ and $\left(u^{c} u^{c} d^{c} \ell^{c}\right)^{2}$, including $n n \rightarrow$ $\pi^{+} \pi^{+} e^{-} e^{-}$, and $p p \rightarrow e^{+} e^{+}$. These are expected to be more suppressed given the high energydimension of the effective operator. We qualitatively estimate that existing experimental bounds on $p p \rightarrow$ $e^{+} e^{+}[5]$ translate into $\Lambda \gtrsim 1 \mathrm{TeV}$.

The $n-\bar{n}$-oscillation bound also outshines the sensitivity of future $\mu^{-} \rightarrow e^{+}$-conversion experiments and cannot be avoided by allowing a non-trivial flavor structure to the new-physics since we are especially interested in firstgeneration quarks. We do note that tree-level BNV processes vanish in the limit $m_{\Sigma \Delta} \rightarrow 0$ and hence can be suppressed if $m_{\Sigma \Delta}$ is smaller than the other mass-scales in the theory. The reason is as follows. If we assign baryon number $+2 / 3$ to $\Sigma$ and $\Delta$ and $\pm 1 / 3$ to $\chi, \chi^{c}$ (in units where the quarks have lepton number $1 / 3$ ), baryon number is violated by the interactions proportional to $y_{\Sigma \alpha}, y_{\Delta \alpha}$-by one unit-and $m_{\Sigma \Delta}$ - by two units. Furthermore, if we assign lepton-number zero to all the new-physics fields, lepton number is violated by $y_{\Sigma \alpha}, y_{\Delta \alpha}$-by one unit. This means that if $m_{\Sigma \Delta}$ is zero $n-\bar{n}$-oscillation requires one to rely on the interactions proportional to $y_{\Sigma \alpha}, y_{\Delta \alpha}$, which also create or destroy leptons. Since there are no leptons in $n-\bar{n}$-oscillation, these interactions contribute to it only at the loop level. In this case, we still expect strong bounds on $\Lambda \gtrsim 100 \mathrm{TeV}$, similar to the one-loop contribution discussed in the next model (model $\psi \Delta \Phi)$ ). These can be ameliorated by judiciously assuming a subset of newphysics couplings is small.

\section{Model $\psi \Delta \Phi \Phi$}

Here, the SM particle content is augmented by a couple of colored vectorlike quarks $\psi \sim(\overline{3}, 1)_{4 / 3}$ and $\psi^{c} \sim$ $(3,1)_{-4 / 3}$, a colored exotic scalar $\Delta \sim(6,1)_{-2 / 3}$, and a doubly-charged scalar $\Phi \sim(1,1)_{-2}$. The Lagrangian is given by 


$$
\begin{aligned}
\mathcal{L}_{\psi \Delta \Phi}= & \mathcal{L}_{\mathrm{SM}}+\mathcal{L}_{\text {kin }}+y_{\Phi \alpha \beta} \Phi \ell_{\alpha}^{c} \ell_{\beta}^{c}+y_{\Delta d} \Delta d^{c} d^{c} \\
& +y_{\Phi \psi} \bar{\Phi} \psi^{c} u^{c}+y_{\Delta \psi} \Delta \psi u^{c}+m_{\psi} \psi \psi^{c} \\
& +V(\Phi, 0, \Delta)+\text { H.c. }
\end{aligned}
$$

where $\mathcal{L}_{\mathrm{SM}}$ is the SM Lagrangian, $\mathcal{L}_{\text {kin }}$ contains the kineticenergy terms for the new particles, and $V(\Phi, 0, \Delta)$ is the most general scalar potential involving the scalars $\Delta, \Phi$, written out explicitly in Appendix A.

It is easy to check that this model realizes $\mathcal{O}_{s}^{\alpha \beta}$ via topology 2 [Fig. 5(right)] and

$$
\frac{g_{\alpha \beta}}{\Lambda^{5}} \equiv \frac{y_{\Phi \alpha \beta} y_{\Phi \psi} y_{\Delta \psi} y_{\Delta d}^{*}}{M_{\Phi}^{2} M_{\Delta}^{2} m_{\psi}}
$$

Here, like in model $\zeta \Phi \Sigma, y_{\Phi \alpha \beta}$ controls the lepton-flavor structure of the model. $\mu^{-} \rightarrow e^{+}$-conversion rates are proportional to $\left|y_{\Phi e \mu}\right|^{2}$, while those for $0 \nu \beta \beta$ are proportional to $\left|y_{\text {Фee }}\right|^{2}$.

As far as CLFV is concerned, this model is very similar to model $\zeta \Phi \Sigma$ since here and there the presence of the doubly-charged scalar $\Phi$ determines most of the leptonnumber conserving phenomenology. Similar to model $\zeta \Phi \Sigma$, the CLFV bounds can be avoided by assuming the new-physics couplings are not generic. If the new-physics portion of the Lagrangian respects an $L_{\mu}-L_{e}$ global symmetry, all CLFV bounds vanish to a very good approximation.

Like model $\chi \Delta \Sigma$, here baryon number is violated but, also like model $\chi \Delta \Sigma$, there is a $Z_{2}$ "lepton-parity"-all lepton-fields are odd and all other fields are even-which implies baryon decays into leptons are not allowed. If we assign lepton-number +2 to $\Phi$, baryon-number $+2 / 3$ to $\Delta$, and baryon-number $\mp 1 / 3$ to $\psi, \psi^{c}$, baryon-numberviolating phenomena are proportional to the $\lambda_{\bar{\Delta} \Phi}$ coupling in the scalar potential. The same coupling also violates lepton-number by two units. ${ }^{5}$ This implies that $n-\bar{n}$ oscillations do not occur at the tree level since BNV is always accompanied by LNV. However, at oneloop, $n-\bar{n}$-oscillations can take place, as depicted in the Feynman diagram in Fig. 10. It translates into the effective Lagrangian

$$
\mathcal{L}_{n-\bar{n}}=\frac{y_{\Delta d}^{2} y_{\Phi \psi} y_{\Delta \psi} \lambda_{\bar{\Delta} \Phi} m_{\Psi}}{16 \pi^{2} M_{\Delta}^{4} \Lambda^{2}}\left(u^{c} d^{c} d^{c}\right)^{2},
$$

where $\Lambda$ is an effective scalar arising out of the masses of $\Delta$, $\Phi$ and $\psi$. Assuming all couplings are $\mathcal{O}(1)$ and all mass scales are of the same order, current experimental bounds translate into

\footnotetext{
${ }^{5}$ From this perspective, the $y_{\Phi \psi}$-coupling violates lepton number by two units.
}

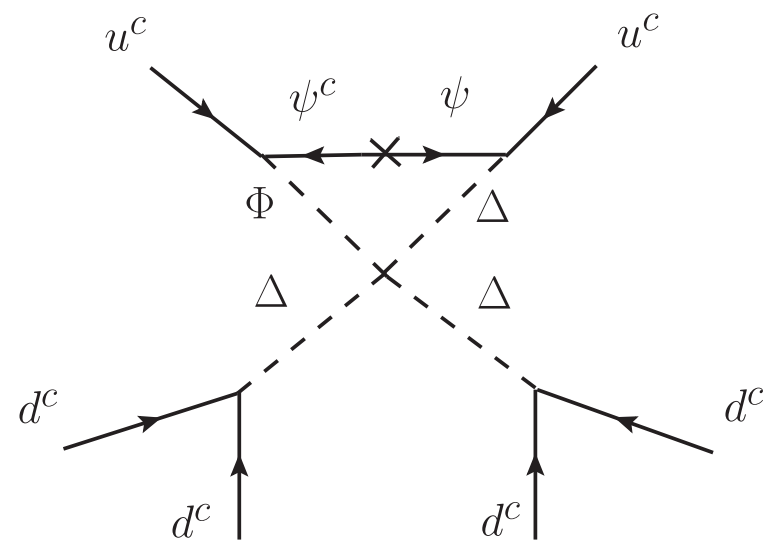

FIG. 10. One-loop Feynman diagram that mediates $n-\bar{n}$ oscillations in model $\psi \Delta \Phi$.

$$
\Lambda \gtrsim 127 \mathrm{TeV}
$$

As advertised, however, baryon-number violation is proportional to $\lambda_{\bar{\Delta} \Phi}$ and can be suppressed-or eliminated completely-in the limit $\lambda_{\bar{\Delta} \Phi} \rightarrow 0$, when baryon number is a good symmetry of the Lagrangian.

As in model $\chi \Delta \Sigma$, here one can also construct the dimensional-twelve operator $\left(d^{c} d^{c} d^{c} \overline{\ell^{c}}\right)^{2}$ which gives rise to phenomena like $n n \rightarrow \pi^{+} \pi^{+} e^{-} e^{-}$. Such processes are higher dimensional, and hence expected to be more strongly suppressed. A subset of the bounds, estimated here and in the previous subsubsections, are summarized in Fig. 11.

\section{Model $\Phi \mathbf{\Phi} \Delta$}

Here, the SM particle content is augmented by only scalar fields: a color-singlet doubly-charged scalar $\Phi \sim(1,1)_{-2}$, and two colored scalars, $\Sigma \sim(6,1)_{4 / 3}$ and $\Delta \sim(6,1)_{-2 / 3}$. The most general renormalizable Lagrangian is

$$
\begin{aligned}
\mathcal{L}_{\Phi \Sigma \Delta}= & \mathcal{L}_{\mathrm{SM}}+\mathcal{L}_{\text {kin }}+y_{\Phi \alpha \beta} \Phi \ell_{\alpha}^{c} \ell_{\beta}^{c}+y_{\Delta d} \Delta d^{c} d^{c} \\
& +y_{\Sigma u} \Sigma u^{c} u^{c}+V(\Phi, \Sigma, \Delta)+\text { H.c. }
\end{aligned}
$$

where $\mathcal{L}_{\mathrm{SM}}$ is the SM Lagrangian, $\mathcal{L}_{\text {kin }}$ contains the kineticenergy terms for the new particles, and $V(\Phi, \Sigma, \Delta)$ is the most general scalar potential involving the scalars $\Phi, \Delta, \Sigma$, written out explicitly in Appendix A.

This is the only no-vectors model where the effective operator $O_{s}^{\alpha \beta}$ is realized at the tree level through topology 1 , and the effective scale is given by

$$
\frac{g_{\alpha \beta}}{\Lambda^{5}} \equiv \frac{y_{\Phi \alpha \beta} y_{\Sigma u} y_{\Delta d}^{*} m_{\Delta \Sigma \Phi}^{*}}{M_{\Phi}^{2} M_{\Delta}^{2} M_{\Sigma}^{2}}
$$

Here, like in model $\zeta \Phi \Sigma$ and model $\psi \Delta \Phi, y_{\Phi \alpha \beta}$ controls the lepton-flavor structure of the model. $\mu^{-} \rightarrow e^{+}$-conversion 


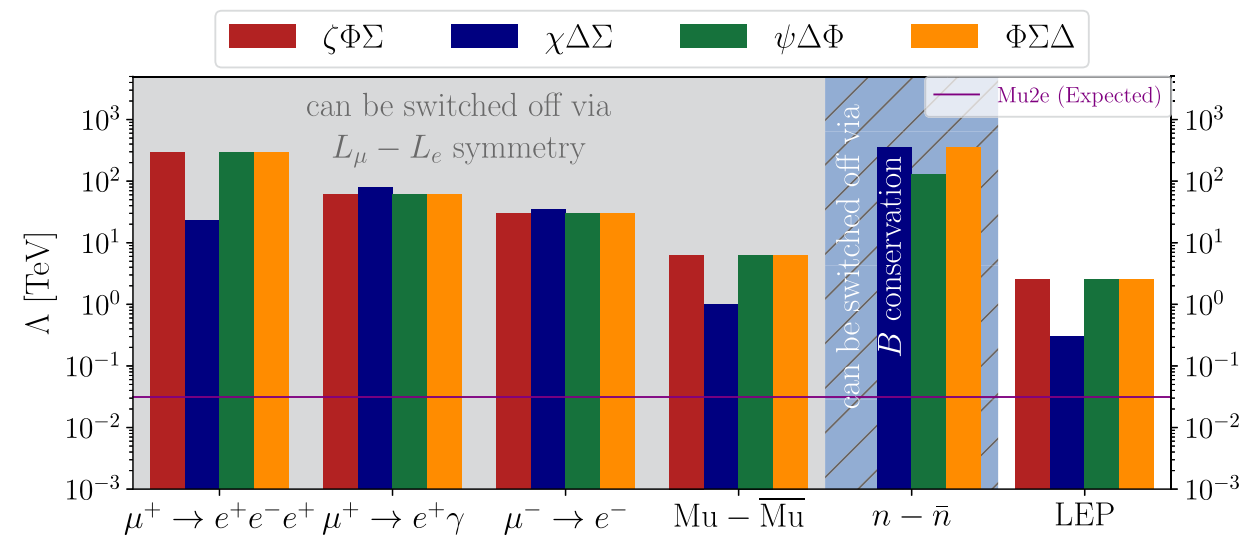

FIG. 11. Summary of the most stringent CLFV, BNV, and lepton-scattering bounds on the effective scale of the all-singlets operator for the different models discussed in the text. These bounds assume that all new physics couplings are of order one and all new physics masses are approximately the same. Other bounds are discussed in the text. Bounds from the observables in the grey area can be softened or eliminated if the new-physics couplings have a very non-generic lepton-flavor structure (e.g., if the new-physics model obeys, at least approximately, an $L_{\mu}-L_{e}$ symmetry, as discussed in the text). Bounds from $n-\bar{n}$-oscillations, in the blue area, can be softened or eliminated if the new-physics couplings are chosen in a way that baryon number is at least approximately conserved. Note that model $\zeta \Phi \Sigma$ conserves baryon number and hence does not contribute to $n-\bar{n}$ oscillations. In the limit where the masses of the new particles are heavy, there are independent hadron collider bounds similar to those from LEP. The expected sensitivity of the Mu2e experiment is indicated by the solid line.

rates are proportional to $\left|y_{\Phi e \mu}\right|^{2}$, while those for $0 \nu \beta \beta$ are proportional to $\left|y_{\Phi e e}\right|^{2}$. The CLFV phenomenology here is very similar to the one in model $\zeta \Phi \Sigma$ and model $\psi \Delta \Phi$.

If we choose to assign lepton-number +2 to $\Phi$ and baryon-number $+2 / 3$ to both $\Sigma$ and $\Delta$, all LNV and BNV couplings are in the scalar potential. Some couplings violate only baryon number (e.g., $m_{\Sigma \Delta}$ ), some violate only lepton number (e.g., $\left.m_{\Delta \Sigma \Phi}\right)$, ${ }^{6}$ while others violate both (e.g., $\left.\lambda_{\bar{\Delta} \Phi}\right)$. This means that BNV phenomena can occur at the tree level, like in model $\chi \Delta \Sigma$. Indeed, $n-\bar{n}$-oscillations occur at the tree level via the Feynman diagram in Fig. 9.

A subset of the bounds, estimated in the previous subsubsections, are summarized in Fig. 11. Here too BNV phenomena are controlled by a different set of couplings as LNV ones, and can be "turned off" by imposing baryon number as a conserved, or approximately conserved, symmetry.

\section{B. Models with a new vector boson}

As discussed earlier and summarized in Table IV, there are two different vector bosons capable of realizing the all-singlets operator at the tree level in a way that other LNV operators are also avoided. These are a color-singlet with hyper-charge one $\left[(1,1)_{1}\right]$ or a color-octet with hypercharge one $\left[(8,1)_{1}\right]$. We will refer to both of them as $C_{\mu}$. The only allowed couplings of $C^{\mu}$ to SM fermions is $C_{\mu} \overline{d^{c}} \bar{\sigma}^{\mu} u^{c}$ (see Table IV). If, however, left-handed

\footnotetext{
${ }^{6}$ Note that the effective coupling of $O_{s}^{\alpha \beta}$, Eq. (3.32), is proportional to $m_{\Delta \Sigma \Phi}$.
}

antineutrino fields $\nu^{c}$ exist, the following coupling is also allowed, for the color-singlet $C^{\mu}: C_{\mu} \overline{\ell^{c}} \bar{\sigma}^{\mu} \nu^{c}$. We return to the issue of generating neutrino masses in Sec. VI.

Quantum field theories with massive vector bosons, in general, have severe problems in the ultraviolet. The models presented here are no exception. The most general "UV-complete" Lagrangians we will be considering are, in fact, not really UV-complete as, for example, we expect the scattering of longitudinal vector bosons to violate partialwave unitarity in the ultraviolet, indicating that a proper UV-completion of the theory is required. As is well known, there are a few possible ways to UV-complete theories with massive vector bosons. They could, for example, be composite objects of some confining gauge theory. In the scenarios discussed here, since the vector-boson $C^{\mu}$ carries electric-charge (and hypercharge) and, in some cases, color, some of the fundamental fields of the UV theory must transform nontrivially under the SM gauge symmetry. Another possibility is that $C^{\mu}$ is a gauge boson associated to some broken gauge symmetry. The fact that $C^{\mu}$ is charged and potentially colored makes the construction of UV-complete models nontrivial. Below-in model $\mathrm{NC}$-we explore in a little more detail the possibility that the color-singlet $C^{\mu}$ may be the $W_{R}$-boson in left-right symmetric extensions of the SM.

All models are listed below. It turns out that, unlike the no-vectors models, all of them conserve baryon number. Phenomenologically, most of the models give rise to the CLFV processes already discussed before and the bounds and challenges one needs to address are very similar to those of no-vectors models. For this reason, we do not 
elaborate on experimental bounds but, for the most part, concentrate on whatever unique features the different models possess.

\section{Models $\Phi C$}

Here, the SM particle content is augmented by a chargedscalar $\Phi \sim(1,1)_{-2}$, and a vector $C^{\mu} \sim(8,1)_{1}$. The most general renormalizable Lagrangian is

$$
\begin{aligned}
\mathcal{L}_{\Phi C}= & \mathcal{L}_{\mathrm{SM}}+\mathcal{L}_{\text {kin }}+y_{\Phi \alpha \beta} \Phi \ell_{\alpha}^{c} \ell_{\beta}^{c}+g_{C d u} C_{\mu} \overline{d^{c}} \bar{\sigma}^{\mu} u^{c} \\
& +V(\Phi, C)+\text { H.c. },
\end{aligned}
$$

where $\mathcal{L}_{\mathrm{SM}}$ is the SM Lagrangian, $\mathcal{L}_{\text {kin }}$ contains the kineticenergy terms for the new particles, and $V(\Phi, C)$ is the vector-scalar potential listed in Eq. (A2) in Appendix A. This is the simplest model as far as its particle content is concerned. Lepton number can be assigned to the various fields in a way that the term $C_{\mu} C^{\mu} \Phi$ in the vector-scalar potential violates it by two units ( $\Phi$ lepton-number $2, C^{\mu}$ lepton-number zero).

The all-singlets operator is realized at the tree level via topology 1 . The effective couplings and scale are

$$
\frac{g_{\alpha \beta}}{\Lambda^{5}} \equiv \frac{y_{\Phi \alpha \beta} g_{C d u}^{2} m_{C \Phi}^{*}}{M_{\Phi}^{2} M_{C}^{4}} .
$$

Here, like all models that include the $\Phi$-field, $y_{\Phi \alpha \beta}$ controls the lepton-flavor structure of the model. $\mu^{-} \rightarrow e^{+}-$ conversion rates are proportional to $\left|y_{\Phi e \mu}\right|^{2}$, while those for $0 \nu \beta \beta$ are proportional to $\left|y_{\Phi e e}\right|^{2}$.

A very similar Lagrangian describes the model where the gauge boson is a color-singlet, $C_{\mu} \sim(1,1)_{1}$. The only difference is the presence of an extra interaction between $C^{\mu}$ and the Higgs doublet, proportional to $\bar{C}_{\mu} H D^{\mu} H$. This interaction is inconsequential for LNV.

There are strong constraints on the production of charged vector bosons that couple to quarks, which will be discussed later, while, as already mentioned, the colorsinglet vector also allows couplings to left-handed antineutrinos $\propto C_{\mu} \bar{\ell}^{c} \bar{\sigma}^{\mu} \nu^{c}$.

\section{Model $\zeta \Phi C$ and $\psi \Phi C$}

We can add a new vectorlike fermion to model $\Phi C$ in such a way that more LNV interactions are allowed and one generates, at the tree level, the all-singlets operator via both topologies in Fig. 5. This can be done in two different ways.

We can add to the particle content of model $\Phi C$ a pair of vectorlike quarks $\zeta \sim(\overline{3}, 1)_{-5 / 3}$ and $\zeta^{c} \sim(3,1)_{5 / 3}$. The most general Lagrangian is, assuming $C^{\mu}$ is a color-octet vector-boson,

$$
\begin{aligned}
\mathcal{L}_{\zeta \Phi C}= & \mathcal{L}_{\mathrm{SM}}+\mathcal{L}_{\mathrm{kin}}+y_{\Phi \alpha \beta} \Phi \ell_{\alpha}^{c} \ell_{\beta}^{c}+g_{C d u} C_{\mu} \overline{d^{c}} \bar{\sigma}^{\mu} u^{c} \\
& +y_{\Phi \zeta^{c}} \Phi \zeta^{c} d^{c}+g_{C u \zeta} C_{\mu} \overline{u^{c}} \bar{\sigma}^{\mu} \zeta+m_{\zeta} \zeta \zeta^{c} \\
& +V(\Phi, C)+\text { H.c. },
\end{aligned}
$$

where $\mathcal{L}_{\mathrm{SM}}$ is the SM Lagrangian, $\mathcal{L}_{\text {kin }}$ contains the kineticenergy terms for the new particles, and $V(\Phi, C)$ is the vector-scalar potential listed in Eq. (A2) in Appendix A. The coefficient of the all-singlets operator is

$$
\frac{g_{\alpha \beta}}{\Lambda^{5}} \equiv \frac{y_{\Phi \alpha \beta} g_{C d u}^{2} m_{C \Phi}^{*}}{M_{\Phi}^{2} M_{C}^{4}}+\frac{y_{\Phi \alpha \beta} g_{C d u} g_{C u \zeta}^{*} y_{\Phi \zeta^{c}}^{*}}{M_{\Phi}^{2} M_{C}^{2} m_{\zeta}} .
$$

Instead, we could add to the particle content of model $\Phi C$ a pair of vectorlike quarks $\psi \sim(\overline{3}, 1)_{4 / 3}$ and $\psi^{c} \sim(3,1)_{-4 / 3}$. The most general Lagrangian in this case is, assuming $C^{\mu}$ is a color-octet vector-boson,

$$
\begin{aligned}
\mathcal{L}_{\psi \Phi C}= & \mathcal{L}_{\mathrm{SM}}+\mathcal{L}_{\mathrm{kin}}+y_{\Phi \alpha \beta} \Phi \ell_{\alpha}^{c} \ell_{\beta}^{c}+g_{C d u} C_{\mu} \bar{d}^{c} \bar{\sigma}^{\mu} u^{c} \\
& +y_{\Phi \psi} \bar{\Phi} \psi^{c} u^{c}+g_{C \psi d} C_{\mu} \bar{\psi} \bar{\sigma}^{\mu} d^{c}+m_{\psi} \psi \psi^{c} \\
& +V(\Phi, C)+\text { H.c. }
\end{aligned}
$$

where $\mathcal{L}_{\mathrm{SM}}$ is the SM Lagrangian, $\mathcal{L}_{\text {kin }}$ contains the kineticenergy terms for the new particles, and $V(\Phi, C)$ is the vector-scalar potential listed in Eq. (A2) in Appendix A. Clearly, this is very similar to the model in Eq. (3.35), with just the charges for the vectorlike quarks different. Here, the coefficient of the all-singlets operator is

$$
\frac{g_{\alpha \beta}}{\Lambda^{5}} \equiv \frac{y_{\Phi \alpha \beta} g_{C d u}^{2} m_{C \Phi}^{*}}{M_{\Phi}^{2} M_{C}^{4}}+\frac{y_{\Phi \alpha \beta} g_{C d u} g_{C \psi d}^{*} y_{\Phi \psi}}{M_{\Phi}^{2} M_{C}^{2} m_{\psi}} .
$$

In both scenarios one can assign lepton number to the new-physics fields such that both $m_{C \Phi}$ and the coupling of the $\Phi$ field to the new fermion and a quark- $y_{\Phi \zeta^{c}}$ or $y_{\Phi \psi}-$ violate lepton number by two units. In this way, one can control which topology contributes most to the all-singlets operator. Note, however, that both contributions to $g_{\alpha \beta} / \Lambda^{5}$ are proportional to $y_{\Phi \alpha \beta} /\left(M_{\Phi}^{2} M_{C}^{2}\right)$.

\section{Model NC}

The new vector-boson $C_{\mu} \sim(8,1)_{1}$ can also be used to generate the all-singlets operator at the tree level if there are color-octet fermions $N \sim(8,1)_{0}$. In this case, the most general renormalizable Lagrangian is

$$
\begin{aligned}
\mathcal{L}_{N C}= & \mathcal{L}_{\mathrm{SM}}+\mathcal{L}_{\text {kin }}+g_{C N \alpha} C_{\mu} \overline{\ell_{\alpha}^{c}} \bar{\sigma}^{\mu} N+g_{C d u} C_{\mu} \overline{d^{c}} \bar{\sigma}^{\mu} u^{c} \\
& +m_{N} N N+V(0, C)+\text { H.c },
\end{aligned}
$$

where $\mathcal{L}_{\mathrm{SM}}$ is the SM Lagrangian, $\mathcal{L}_{\text {kin }}$ contains the kineticenergy terms for the new particles, and $V(0, C)$ is the potential for the vector field listed in Eq. (A2) in 
Appendix A. One can assign lepton number to the new fields, -1 for $N$, zero for $C_{\mu}$, such that the Majorana masses of the color-octet fermions control LNV. The operator $O_{s}^{\alpha \beta}$ is generated at the tree level-topology 2-and its coefficient is

$$
\frac{g_{\alpha \beta}}{\Lambda^{5}} \equiv \frac{g_{C d u}^{2} g_{C N \alpha}^{*} g_{C N \beta}^{*}}{M_{C}^{4} m_{N}} .
$$

Here, the lepton-flavor structure of the all-singlets operator is governed by the couplings $g_{C N \alpha}$. The $\mu^{-} \rightarrow e^{+}$conversion rates are proportional to $\left|g_{C N e} g_{C N \mu}\right|^{2}$, while those for $0 \nu \beta \beta$ are proportional to $\left|g_{C N e}^{2}\right|^{2}$.

Similar to many of the previous models, CLFV process are ubiquitous here. However, since $\mu^{c}$ and $e^{c}$ couple to the same fields through the operators $C_{\mu} \overline{\ell^{c}} \bar{\sigma}^{\mu} N$, and since the rate for $\mu^{-} \rightarrow e^{+}$-conversion requires both $g_{C N e}, g_{C N \mu}$ to be relevant, it is not possible to choose new physics couplings such that most CLFV observables are relatively suppressed. In this scenario, given several existing experimental constraints, the rates for $\mu^{-} \rightarrow e^{+}$-conversion are outside the reach of the next-generation experiments. However, it is possible to slightly modify the model to suppress CLFV. Instead of introducing one field $N$, one can introduce the pair $N$ and $N^{c}$ with the $L_{\mu}-L_{e}$ charges +1 and -1 respectively; in other words, the Lagrangian will include terms $C_{\mu} \overline{e^{c}} \bar{\sigma}^{\mu} N$ and $C_{\mu} \overline{\mu^{c}} \bar{\sigma}^{\mu} N^{c}$. The Majorana mass term would be forbidden by the global symmetry and replaced with the Dirac mass term proportional to $N N^{c}$.

A similar scenario arises with $C_{\mu} \sim(1,1)_{1}$ and a gaugesinglet fermion $N \sim(1,1)_{0}$. In this case, a neutrino Yukawa interaction $L H N$ is also allowed and the model is nothing more than the type-I seesaw model [54-59] plus a chargeone vector boson. This scenario violates the requirements we introduced earlier: here, the Weinberg operator $(L H)^{2}$ is generated at the tree level, as in the type-I seesaw model. It should be pointed out that it is possible to suppress the tree-level contribution to the Weinberg operator by choosing very small neutrino Yukawa couplings. In this case, the phenomenology is similar to the one discussed in the previous models. ${ }^{7}$

As discussed before, models with a heavy vector-boson require extra care in order to be rendered consistent in the ultraviolet. In the case of $C_{\mu} \sim(1,1)_{1}$, this can be achieved by appreciating that it acts like the right-handed W-boson $W_{R}$ in left-right symmetric models [58,60-63]. In fact, the Lagrangian for $C_{\mu} \sim(1,1)_{1}$ and the gauge-singlet fermion $N \sim(1,1)_{0}$ is a subset of the left-right symmetric Lagrangian, where the SM gauge group is extended to

\footnotetext{
${ }^{7}$ Here, dimension-seven operators like $L_{\mu} H \overline{e^{c}} \overline{u^{c}} d^{c}$ are also generated. These yield large contributions to neutrino masses if the Yukawa couplings are not small. In the case of the color-octet Majorana fermion $N \sim(8,1)_{0}$, Yukawa couplings to the charged leptons do not exist and therefore this is not an issue.
}

TABLE V. Fields in model $N C$ assuming $C_{\mu}$ is the right-handed $W_{R}$-boson of an $S U(2)_{L} \times S U(2)_{R} \times U(1)_{B-L}$ gauge theory.

\begin{tabular}{lc}
\hline \hline Particles & $\left(\mathrm{SU}(2)_{L}, \mathrm{SU}(2)_{R}, \mathrm{U}(1)_{B-L}\right)$ \\
\hline$Q_{L} \equiv\left(u_{L}, d_{L}\right)$ & $(2,1,1 / 6)$ \\
$Q_{R} \equiv\left(u_{R}, d_{R}\right)$ & $(1,2,1 / 6)$ \\
$\psi_{L} \equiv\left(\nu_{L}, e_{L}\right)$ & $(2,1,-1 / 2)$ \\
$\psi_{R} \equiv\left(\nu_{R}, e_{R}\right)$ & $(1,2,-1 / 2)$ \\
$\Delta_{L} \equiv$ scalar & $(3,1,1)$ \\
$\Delta_{R} \equiv$ scalar & $(1,3,1)$ \\
$\Phi_{L R} \equiv$ scalar & $\left(2,2^{*}, 0\right)$ \\
\hline \hline
\end{tabular}

$S U(2)_{L} \times S U(2)_{R} \times U(1)_{B-L}$. This model requires an extended Higgs sector to break the $S U(2)_{R} \times U(1)_{B-L} \rightarrow$ $U(1)_{Y}$. To avoid bounds from collider experiments, this breaking needs to happen at a higher scale so that the new gauge bosons $W_{R}, Z_{R}$ s have large-enough mass. Candidate charge-assignments of the particles under $\mathrm{SU}(2)_{L} \times$ $\mathrm{SU}(2)_{R} \times \mathrm{U}(1)_{B-L}$ are listed in Table $\mathrm{V}$, where we associate $N$ to the conjugate of the right-handed neutrino $\nu_{R}$.

The vev of $\Delta_{R}$, the $S U(2)_{R}$ scalar triplet, gives Majorana masses to the right-handed neutrinos, while that of the of the $S U(2)_{L}$ scalar triplet $\Delta_{L}$ contributes to the Majorana masses of the left-handed neutrinos. One can construct Yukawa interactions involving the Higgs bidoublet $\Phi_{L R}$, which leads to the $L H N$ Yukawa interaction. In this ana$\log y, C^{\mu} \equiv W_{R}^{\mu+}$, and the interactions $C_{\mu} \bar{\ell}^{c} \bar{\sigma}^{\mu} N, C_{\mu} \bar{d}^{c} \bar{\sigma}^{\mu} u^{c}$ are gauge interactions.

\section{COLLIDER BOUNDS}

Here we briefly discuss interesting signatures and constraints we expect from collider experiments; a detailed collider study of all models listed in the previous section is beyond the scope of this paper. All new physics particles introduced in the different models are listed in Table IV. They include colored vectorlike fermions, charged and colored scalars, and charged and colored vector-bosons.

As mentioned earlier, the $\Phi$-scalar will mediate $e^{+} e^{-} \rightarrow$ $e^{+} e^{-}$or $e^{+} e^{-} \rightarrow \mu^{+} \mu^{-}$in the $t$-channel. In the limit where the $\Phi$ mass is larger than the center-of-mass energy of the collider, these interactions are already constrained by measurements at LEP [44]. For lighter masses, different, stringent constraints on the new-physics couplings are expected. Future $e^{+} e^{-}$colliders under consideration, like the ILC [64], FCC-ee [65], and CEPC [66], would be sensitive to much higher effective mass-scales. The ILC, for example, with an integrated luminosity of $1000 \mathrm{fb}^{-1}$, is capable of probing new physics scales $\Lambda$ that are roughly below $75 \mathrm{TeV}[64,67]$ (or $M_{\Phi} \lesssim 20 \mathrm{TeV}$ for order one couplings) through the process $e^{+} e^{-} \rightarrow \mu^{+} \mu^{-}$. The exact sensitivity would depend on the polarization of the electron and positron beams as well as systematic uncertainties at the ILC. An $e^{-} e^{-}$collider would be sensitive to $\Phi$ $s$-channel exchange and the properties of $\Phi$ could be 
studied-or constrained-on-resonance if the collider energy were high enough.

The colored scalars $\Sigma$ and $\Delta$, and $C_{\mu}$ (both the color-singlet and the color-octet) can be produced at hadron colliders like the LHC through the quark or the gluon channels. For example, the dijet channel $q q \rightarrow \Sigma(\Delta) \rightarrow q q$ can be used to probe the contact interaction $\left(y^{2} / M_{\Sigma(\Delta)}^{2}\right) q^{c} q^{c} \overline{q^{c}} \overline{q^{c}}$, which are a valid description of colored-scalar exchange in the limit where the scalar masses are beyond the reach of the collider. Recent dijet studies at ATLAS and CMS $[5,68,69]$ translate into a lower bound on the mass of scalar diquarks [70] and, in our case, imply masses for $\Sigma, \Delta$, and $C_{\mu}$ that exceed around $5 \mathrm{TeV}$, for order one couplings. $\Sigma$ and $\Delta$ will also mediate, at the tree level, processes like $g g \rightarrow \Sigma \Sigma(\Delta \Delta) \rightarrow 4 q$. The corresponding signature is a pair of dijet resonances and can be used to constrain the properties of the colored scalars. The corresponding bounds, however, are expected to be weaker than those of dijet searches as long as the couplings between the new bosons and the quarks are order one. Note that the few $\mathrm{TeV}$ upper bound does not trivially apply for smaller couplings and lower masses for $\Sigma$ and $\Delta$ and $C_{\mu}$, see, for example, $[68,69]$. Relatively light bosons that couple to quarks relatively strongly are know to survive collider constraints, see e.g., [71]. A detailed analysis of this very rich topic, as mentioned above, is beyond the scope of this paper.

The literature on searches for vectorlike exotic quarksincluding octet "neutrinos"-is also large and diverse. Bounds, many of which are listed and briefly discussed in the particle data book [5], hover around $500 \mathrm{GeV}$. A more detailed discussion of exotic quark searches in the LHC can be found, for example, in [72]. Existing bounds depend rather strongly on the decay properties of the exotic colored fermions. Model-independent bounds are much weaker, as summarized, for example, in [5].

New colored (and/or charged) particles that couple to the SM Higgs boson will modify the Higgs production rate via gluon fusion and the decay rate into two photons, i.e., $g g \rightarrow H \rightarrow \gamma \gamma$. The doubly-charged scalar $\Phi$ also contributes to the decay process $H \rightarrow 4 \ell$ at tree level. Precision measurements of Higgs production and decay will translate into bounds on the properties of $\Phi, \Delta, \Sigma$, and $C_{\mu}$. In addition, one should also worry about electroweak precision tests of the SM, although corresponding constraints might be weaker than direct searches at the LHC. The scalar $\Phi$ and the vector $C_{\mu}$, for example, will modify (via triangle loop diagrams) the partial decay widths of the $Z$-boson into quarks and leptons. Moreover, if $\Phi$ only couples to the pair $e \mu$, the universality of $Z \rightarrow e e, \mu \mu$ and $\tau \tau$ will be violated. Finally, as all new charged particles listed in Table IV are singlets under $S U(2)_{L}$, there are no contributions to the oblique parameters $(S, T, U)[73,74]$, as demonstrated in, e.g., [75], where contributions from vectorlike down-type quarks to the oblique parameters were shown to vanish if these do not mix with the SM $S U(2)_{L}$ quark doublets.
LNV phenomena can also be probed at colliders. The allsinglets operator will mediate $\overline{u^{c}} \overline{u^{c}} \rightarrow e^{c} e^{c} \overline{d^{c}} \overline{d^{c}}$ scattering, as discussed briefly in [24]. Up to color factors and symmetry factors, the cross section for this process is $\sigma \propto g^{2} s^{4} / \Lambda^{10}$. This can lead to interesting signatures at the large hadron collider (LHC) or the international linear collider (ILC) (exchanging the role of the charged-leptons and the up-quarks). The latter is similar to searches for the LNV process $e^{-} e^{-} \rightarrow W^{-} W^{-}$at lepton colliders, except for the fact that the final-state dijet invariant masses are not related to the $W$-boson mass [76]. Similar studies could also be pursued with a muon collider [77].

\section{SUMMARY: VIABILITY OF LARGE $\mu^{-} \rightarrow e^{+}$ CONVERSION RATES}

Concerning the viability of different models to mediate observable $\mu^{-} \rightarrow e^{+}$-conversion in nuclei at next-generation experiments, our main results, illustrated in Fig. 11 with the assumptions of a universal mass scale $\Lambda$ for new particles and $\mathcal{O}(1)$ couplings, can be summarized as follows:

(1) For all the models considered, CLFV and $0 \nu \beta \beta$ provide the most stringent bounds on the effective scale of the all-singlet operator. These bounds, $\mathcal{O}(10-100) \mathrm{TeV}$, are much stronger than the sensitivity of next-generation $\mu^{-} \rightarrow e^{+}$-conversion experiments, $\mathcal{O}(10) \mathrm{GeV}$. However, depending on the lepton-flavor structure of the models considered, it is possible to avoid most of these constaints. One possibility discussed here is that if the new-physics Lagrangian respects an $L_{\mu}-L_{e}$ (muon-number minus electron-number) global symmetry, then all the CLFV and $0 \nu \beta \beta$ are significantly weakened.

(2) For models which explicitly violate baryon number, the $n-\bar{n}$-oscillation bound- $\mathcal{O}(100) \mathrm{TeV}-$ also outshines the sensitivity of future $\mu^{-} \rightarrow e^{+}$conversion experiments and cannot be avoided by allowing a nontrivial flavor structure to the new physics. Even in these cases, one can get remove these bounds by postulating that baryon number is a global symmetry of the Lagrangian.

(3) The new interactions predicted by the models are also tightly constrained by LEP and other collider experiments, which also probe scales $(\mathcal{O}(1) \mathrm{TeV})$ beyond the sensitivity of future $\mu^{-} \rightarrow e^{+}$-conversion experiments. These bounds cannot be alleviated by taking advantage of symmetry arguments. They can, however, be weakened by judiciously choosing different couplings (mass-scales) to be relatively small (large), as we discuss in the some concrete scenarios.

\section{DISCUSSIONS AND CONCLUDING REMARKS}

Lepton number and baryon number are accidental global symmetries of the classical SM Lagrangian (and 
baryon-number-minus-lepton-number is an accidental global symmetry of the quantum SM Lagrangian). LNV can be probed in a variety of ways, ranging from rare nuclear processes to collider experiments. So far, there is no direct evidence for LNV. Nonzero neutrino masses are often interpreted as evidence for LNV. In most scenarios where this is the case, because neutrino masses are tiny, the rates for LNV processes are way out of the reach of experimental probes of LNV, except for searches for $0 \nu \beta \beta$.

Here, we concentrated on identifying and discussing models where this is not the case and asked whether there are UV-complete models where the rate for $\mu^{-} \rightarrow e^{+}$conversion in nuclei is close to the sensitivity of nextgeneration experiments. All models identified here violate lepton number at energies scales around one $\mathrm{TeV}$ (or lower) and are best constrained by searches for CLFV, BNV, and $0 \nu \beta \beta$. BNV bounds are sometimes strongly correlated, sometimes not, to the LNV physics. LNV scales that are low enough so one approaches the sensitivity of future searches for $\mu^{-} \rightarrow e^{+}$-conversion in nuclei-along with other LNV process we did not discuss, like rare meson decays (e.g., $D^{-} \rightarrow K^{+} \mu^{-} \mu^{-}$)—require a nongeneric, but often easy to impose, lepton-flavor structure for the new physics. In these cases, high-energy hadron and lepton colliders also offer interesting constraints and opportunities for future discovery.

In more detail, we identified all UV-complete models that realize, at low-energies, the all-singlets dimension-nine operator $\mathcal{O}_{s}=e^{c} \mu^{c} u^{c} u^{c} \overline{d^{c}} \overline{d^{c}}$, identified in [19], and do not realize any other LNV effective operator with similar strength. All new particles-scalars, fermions, and vector bosons-are listed in Table IV. Different models consist of the most general renormalizable Lagrangian of the SM plus different combinations of two or three of these particles. Given a concrete Lagrangian, we estimate the rates for and existing constraints from many low-energy observables. The bounds presented here are rough estimates. For the most part, we assume new-physics couplings to be order one, and assume all new mass scales are of the same order.

Given the various bounds estimated here, it is fair to ask whether, for any of the models identified, it is reasonable to assume that the rate for $\mu^{-} \rightarrow e^{+}$-conversion is within reach of next-generation experiments. The answer, we believe, is affirmative as long as the lepton-flavor structure of the model is not generic and, in some cases, if BNV phenomena are more suppressed than naively anticipated, i.e., BNV couplings are relatively small. At face-value, flavorindependent bounds—see, e.g., Fig. 11-appear to be strong enough to render $\mu^{-} \rightarrow e^{+}$-conversion out of experimental reach for the foreseeable future. This need not be the case, for a few reasons. One is that the different bounds usually apply only to the masses of a subset of the newphysics particles, while the coefficient of the all-singlets operator depends on the mass of all new degrees-offreedom. If one saturates all existing bounds carefully, the scale of the all-singlets operator is lower than the strongest lepton-number conserving bounds, depicted in Fig. 11. Another important point is that, for example, the LEP bounds apply to $y^{2} / M^{2}$ in the limit where $M$ is outside the direct reach of LEP. The coefficient of $\mathcal{O}_{s}$, however, is proportional to $y / M^{2}$ [see, for example, Eq. (3.2), proportional to $y_{\Phi \mu e} / M_{\Phi}^{2}$, versus Eq. (3.15)], proportional to $\left.y_{\Phi \mu e}^{2} / M_{\Phi}^{2}\right)$. For smaller coupling and mass and fixed $y^{2} / M^{2}, y / M^{2}$ is relatively larger. Finally, strictly speaking, all estimates here rely on effective theories. For light-enough new particles and smaller couplings, constraints are, in some cases, significantly weaker once translated into the effective scale of the all-singlets operator $\mathcal{O}_{s}$.

All of the scenarios discussed here fail, by design, to explain the observed active neutrino masses. CLFV constraints alone imply that the contribution of these newphysics models to Majorana active neutrino masses are tiny, smaller than what is required by observations by at least two or three orders of magnitude. In order to accommodate large active neutrino masses, more degrees-of-freedom, different from the ones discussed here, need to be added to the SM particle content. One possibility is to postulate that, other than the new-physics that leads to the all-singlets operator at the tree level, there are other sources of LNV, perhaps at a much larger energy scale. The high-scale typeI seesaw, with gauge-singlet fermions $\nu^{c}$ with Majorana masses much larger than the weak scale would do the trick, for example. Most other models constructed to "explain" small active neutrino Majorana masses should also work out fine. In some cases, the two sources of LNV may "interfere," as would be the case of the type-I seesaw with any of the models that contain the color-singlet vector boson $C^{\mu} \sim(1,1)_{1}$.

Another possibility is to postulate that the physics responsible for the all-singlets operator is the only source of LNV. In this case, small neutrino masses can be accommodated by adding gauge-singlet fermions $\nu^{c}$ without a Majorana mass and tiny Yukawa couplings to $L$ and $H$. The absence of the Majorana masses for the left-handed antineutrinos is natural in the t'Hooft sense: if the LNV parameters in the models discussed here vanish, lepton number is a good symmetry of the Lagrangian. In this case, neutrinos are pseudo-Dirac fermions since the left-handed neutrinos and the left-handed antineutrinos both acquire small Majorana masses ${ }^{8}$ on top of the dominant Dirac masses. These scenarios are constrained, quite severely, by solar neutrino experiments—see [78,79]—since they mediate neutrino-oscillation processes with long oscillation

\footnotetext{
${ }^{8}$ In the case of the right-handed neutrinos, their loop-induced Majorana masses are proportional to the neutrino Yukawa couplings. Since the Yukawa couplings are very small, so are the Majorana masses, in spite of the fact that they are associated to a relevant operator.
} 
lengths. A more detailed analysis is beyond the scope of this paper.

In summary, UV models which induce $\mathcal{O}_{s}$ at the treelevel can yield a $\mu^{-} \rightarrow e^{+}$-conversion rate that is accessible to future experiments if (i) the UV physics respects, at least approximately, a lepton-flavor symmetry, such as $L_{\mu}-L_{e}$, in order to avoid LFV constraints, (ii) the UV physics respects, at least approximately, baryon-number conservation, in order to evade BNV bounds, and (iii) the UV model contains relatively small couplings, especially those that govern lepton-flavor-conserving observables, in order to avoid constraints like those from LEP.

\section{ACKNOWLEDGMENTS}

We would like to thank Jeff Berryman and Kevin Kelly for useful discussions. The work of A.dG. was supported in part by DOE Grant No. de-sc0010143. W. C. H. acknowledges funding from the Independent Research Fund Denmark, Grant No. DFF 6108-00623. J. K. would like to thank Claudia Hagedorn and the Department of Physics, Chemistry and Pharmacy, as well as the Study Travel Fund of the Faculty of Science at the University of Southern Denmark, for their support for his visit at Northwestern University. He would also like to thank Northwestern University and the particle theory group for their hospitality during his stay. M. S. acknowledges support from the National Science Foundation, Grant No. PHY-1630782, and to the Heising-Simons Foundation, Grant No. 2017-228. The CP3-Origins centre is partially funded by the Danish National Research Foundation, Grant No. DNRF90.

\section{APPENDIX: SCALAR AND VECTOR-SCALAR POTENTIALS}

The most general potential involving all the Higgs and the new scalars $\Phi \sim(1,1)_{-2}, \Delta \sim(6,1)_{-2 / 3}$ and $\Sigma \sim(6,1)_{4 / 3}$, in the no-vectors models, is given by

$$
\begin{aligned}
V(\Phi, \Sigma, \Delta)= & \mu_{\Phi}^{2}|\Phi|^{2}+\mu_{\Sigma}^{2}|\Sigma|^{2}+\mu_{\Delta}^{2}|\Delta|^{2}+\lambda_{\Phi}|\Phi|^{4} \\
& +\lambda_{\Sigma}|\Sigma|^{4}+\lambda_{\Delta}|\Delta|^{4}+\lambda_{H \Phi}|H|^{2}|\Phi|^{2} \\
& +\lambda_{H \Sigma}|H|^{2}|\Sigma|^{2}+\lambda_{H \Delta}|H|^{2}|\Delta|^{2}+\lambda_{\Phi \Sigma}|\Phi|^{2}|\Sigma|^{2} \\
& +\lambda_{\Phi \Delta}|\Phi|^{2}|\Delta|^{2}+\lambda_{\Sigma \Delta}|\Sigma|^{2}|\Delta|^{2}+m_{\Sigma \Delta} \Sigma \Delta^{2} \\
& +\lambda_{\bar{\Delta} \Phi} \bar{\Delta}^{3} \Phi+m_{\Delta \Sigma \Phi} \bar{\Delta} \Sigma \Phi+\lambda_{\Delta \Sigma \Phi} \Delta \Sigma^{2} \Phi .
\end{aligned}
$$

In the text, we also refer to $V(\Phi, \Sigma, 0), V(0, \Sigma, \Delta)$, and $V(\Phi, 0, \Delta)$. These are given by Eq. (A1) where the field labeled 0 is set to zero.

Similarly, the most general potential in the vector models involving the Higgs, the scalar $\Phi$ and the vector $C^{\mu}$ is

$$
\begin{aligned}
V\left(\Phi, C^{\mu}\right)= & \mu_{\Phi}^{2}|\Phi|^{2}+\mu_{C}^{2}\left|C_{\mu}\right|^{2}+\lambda_{\Phi}|\Phi|^{4}+\lambda_{C}\left|C_{\mu}\right|^{4} \\
& +\lambda_{H \Phi}|H|^{2}|\Phi|^{2}+\lambda_{H C}|H|^{2}\left|C_{\mu}\right|^{2} \\
& +\lambda_{\Phi C}|\Phi|^{2}\left|C_{\mu}\right|^{2}+m_{C \Phi} C_{\mu} C^{\mu} \Phi .
\end{aligned}
$$

In the text, we also refer to $V\left(0, C^{\mu}\right)$. This is given by Eq. (A2) where the $\Phi$ field is set to zero.

Throughout, we assume the parameters of the various scalar and scalar-vector potentials are such that none of the new-physics scalar fields acquire vacuum expectation values.
[1] G. 't Hooft, Symmetry Breaking Through Bell-Jackiw Anomalies, Phys. Rev. Lett. 37, 8 (1976).

[2] F. R. Klinkhamer and N. S. Manton, A saddle point solution in the Weinberg-Salam theory, Phys. Rev. D 30, 2212 (1984).

[3] J. Ellis and K. Sakurai, Search for sphalerons in protonproton xollisions, J. High Energy Phys. 04 (2016) 086.

[4] S. H. H. Tye and S. S. C. Wong, Bloch wave function for the periodic sphaleron potential and unsuppressed Baryon and Lepton number violating processes, Phys. Rev. D 92, 045005 (2015).

[5] M. Tanabashi et al. (Particle Data Group), Review of particle physics, Phys. Rev. D 98, 030001 (2018).

[6] J. Schechter and J. W. F. Valle, Neutrino decay and spontaneous violation of Lepton number, Phys. Rev. D 25, 774 (1982).

[7] A. de Gouvêa and P. Vogel, Lepton flavor and number conservation, and physics beyond the Standard Model, Prog. Part. Nucl. Phys. 71, 75 (2013).
[8] A. de Gouvêa, Neutrino mass models, Annu. Rev. Nucl. Part. Sci. 66, 197 (2016).

[9] J. Schechter and J. W. F. Valle, Neutrinoless double beta decay in $\mathrm{SU}(2) \times \mathrm{U}(1)$ theories, Phys. Rev. D 25, 2951 (1982).

[10] W. Rodejohann, Neutrino-less double beta decay and particle physics, Int. J. Mod. Phys. E 20, 1833 (2011).

[11] J. B. Albert et al. (EXO-200 Collaboration), Search for Majorana neutrinos with the first two years of EXO-200 data, Nature (London) 510, 229 (2014).

[12] A. Gando et al. (KamLAND-Zen Collaboration), Limit on Neutrinoless $\beta \beta$ Decay of ${ }^{136} \mathrm{Xe}$ from the First Phase of KamLAND-Zen and Comparison with the Positive Claim in ${ }^{76} \mathrm{Ge}$, Phys. Rev. Lett. 110, 062502 (2013).

[13] M. Agostini et al. (GERDA Collaboration), GERDA results and the future perspectives for the neutrinoless double beta decay search using ${ }^{76} \mathrm{Ge}$, Int. J. Mod. Phys. A 33, 1843004 (2018). 
[14] P. D. Bolton and F. F. Deppisch, Probing nonstandard lepton number violating interactions in neutrino oscillations, Phys. Rev. D 99, 115011 (2019).

[15] Y. Kuno (COMET Collaboration), A search for muon-toelectron conversion at J-PARC: The COMET experiment, Prog. Theor. Exp. Phys. 2013, 22C01 (2013).

[16] H. Natori (DeeMe Collaboration), DeeMe experiment-An experimental search for a mu-e conversion reaction at J-PARC MLF, Nucl. Phys. B, Proc. Suppl. 248-250, 52 (2014).

[17] L. Bartoszek et al. (Mu2e Collaboration), Mu2e technical design report, arXiv:1501.05241.

[18] J. Kaulard et al. (SINDRUM II Collaboration), Improved limit on the branching ratio of $\mu^{-} \rightarrow e^{+}$conversion on titanium, Phys. Lett. B 422, 334 (1998).

[19] J. M. Berryman, A. de Gouvêa, K. J. Kelly, and A. Kobach, Lepton-number-violating searches for muon to positron conversion, Phys. Rev. D 95, 115010 (2017).

[20] B. Yeo, Y. Kuno, M. Lee, and K. Zuber, Future experimental improvement for the search of lepton-numberviolating processes in the $e \mu$ sector, Phys. Rev. D 96, 075027 (2017).

[21] T. Geib, A. Merle, and K. Zuber, $\mu^{-}-e^{+}$conversion in upcoming LFV experiments, Phys. Lett. B 764, 157 (2017).

[22] T. Geib and A. Merle, $\mu^{-}-e^{+}$Conversion from short-range operators, Phys. Rev. D 95, 055009 (2017).

[23] K. S. Babu and C. N. Leung, Classification of effective neutrino mass operators, Nucl. Phys. B619, 667 (2001).

[24] A. de Gouvêa and J. Jenkins, A survey of Lepton number violation via effective operators, Phys. Rev. D 77, 013008 (2008).

[25] P. W. Angel, N. L. Rodd, and R. R. Volkas, Origin of neutrino masses at the LHC: $\Delta L=2$ effective operators and their ultraviolet completions, Phys. Rev. D 87, 073007 (2013).

[26] F. F. Deppisch, L. Graf, J. Harz, and W.-C. Huang, Neutrinoless double beta decay and the baryon asymmetry of the Universe, Phys. Rev. D 98, 055029 (2018).

[27] S. Weinberg, Baryon and Lepton Nonconserving Processes, Phys. Rev. Lett. 43, 1566 (1979).

[28] I. Esteban, M. C. Gonzalez-Garcia, A. HernandezCabezudo, M. Maltoni, and T. Schwetz, Global analysis of three-flavour neutrino oscillations: synergies and tensions in the determination of $\theta_{2} 3, \delta_{C} P$, and the mass ordering, J. High Energy Phys. 01 (2019) 106.

[29] S. Vagnozzi, E. Giusarma, O. Mena, K. Freese, M. Gerbino, S. Ho, and M. Lattanzi, Unveiling $\nu$ secrets with cosmological data: Neutrino masses and mass hierarchy, Phys. Rev. D 96, 123503 (2017).

[30] N. Aghanim et al. (Planck Collaboration), Planck 2018 results. VI. Cosmological parameters, arXiv:1807.06209.

[31] A. Loureiro et al., On the Upper Bound of Neutrino Masses from Combined Cosmological Observations and Particle Physics Experiments, Phys. Rev. Lett. 123, 081301 (2019).

[32] A. Gando et al. (KamLAND-Zen Collaboration), Search for Majorana Neutrinos near the Inverted Mass Hierarchy Region with KamLAND-Zen, Phys. Rev. Lett. 117, 082503 (2016); Addendum, Phys. Rev. Lett. 117, 109903 (2016).
[33] W. H. Bertl et al. (SINDRUM II Collaboration), A Search for muon to electron conversion in muonic gold, Eur. Phys. J. C 47, 337 (2006).

[34] J. C. Helo, M. Hirsch, T. Ota, and F. A. Pereira dos Santos, Double beta decay and neutrino mass models, J. High Energy Phys. 05 (2015) 092.

[35] G. Anamiati, O. Castillo-Felisola, R. M. Fonseca, J. C. Helo, and M. Hirsch, High-dimensional neutrino masses, J. High Energy Phys. 12 (2018) 066.

[36] U. Bellgardt et al. (SINDRUM Collaboration), Search for the decay $\mathrm{mu}+->\mathrm{e}+\mathrm{e}+\mathrm{e}-$, Nucl. Phys. B299, 1 (1988).

[37] Y. Kuno and Y. Okada, Muon decay and physics beyond the standard model, Rev. Mod. Phys. 73, 151 (2001).

[38] M. Fael and C. Greub, Next-to-leading order prediction for the decay $\mu \rightarrow e\left(e^{+} e^{-}\right) \nu \bar{\nu}$, J. High Energy Phys. 01 (2017) 084.

[39] N. Berger (Mu3e Collaboration), The Mu3e experiment, Nucl. Phys. B, Proc. Suppl. 248-250, 35 (2014).

[40] A. M. Baldini et al. (Mu3e Collaboration), Search for the lepton flavour violating decay $\mu^{+} \rightarrow \mathrm{e}^{+} \gamma$ with the full dataset of the MEG experiment, Eur. Phys. J. C 76, 434 (2016).

[41] M. Raidal and A. Santamaria, Muon electron conversion in nuclei versus $\mathrm{mu} \rightarrow \mathrm{e}$ gamma: An effective field theory point of view, Phys. Lett. B 421, 250 (1998).

[42] A. M. Baldini et al., MEG upgrade proposal, arXiv:1301 .7225 .

[43] L. Willmann et al., New Bounds from a Search for Muonium to Antimuonium Conversion, Phys. Rev. Lett. 82, 49 (1999).

[44] t. S. Electroweak (LEP, ALEPH, DELPHI, L3, OPAL, LEP Electroweak Working Group, SLD Electroweak Group, and SLD Heavy Flavor Group Collaborations), A combination of preliminary electroweak measurements and constraints on the Standard Model, arXiv:hep-ex/ 0312023.

[45] G. W. Bennett et al. (Muon g-2 Collaboration), Final report of the Muon E821 anomalous magnetic moment measurement at BNL, Phys. Rev. D 73, 072003 (2006).

[46] M. Davier, A. Hoecker, B. Malaescu, and Z. Zhang, Reevaluation of the hadronic contributions to the Muon g-2 and to $\alpha\left(M_{Z}^{2}\right)$, Eur. Phys. J. C 71, 1515 (2011); Erratum, Eur. Phys. J. C 72, 1874(E) (2012).

[47] K. Hagiwara, R. Liao, A. D. Martin, D. Nomura, and T. Teubner, $(g-2)_{\mu}$ and $\alpha\left(M_{Z}^{2}\right)$ re-evaluated using new precise data, J. Phys. G 38, 085003 (2011).

[48] N. Chakrabarty, C.-W. Chiang, T. Ohata, and K. Tsumura, Charged scalars confronting neutrino mass and muon $g-2$ anomaly, J. High Energy Phys. 12 (2018) 104.

[49] S. R. Moore, K. Whisnant, and B.-L. Young, Second order corrections to the Muon anomalous magnetic moment in alternative electroweak models, Phys. Rev. D 31, 105 (1985).

[50] M. Lindner, M. Platscher, and F. S. Queiroz, A call for new physics: The muon anomalous magnetic moment and lepton flavor violation, Phys. Rep. 731, 1 (2018).

[51] J. Grange et al. (Muon g-2 Collaboration), Muon (g-2) technical design report, arXiv:1501.06858. 
[52] M. Baldo-Ceolin et al., A new experimental limit on neutron-anti-neutron oscillations, Z. Phys. C 63, 409 (1994).

[53] D. G. Phillips, II et al., Neutron-antineutron oscillations: Theoretical status and experimental prospects, Phys. Rep. 612, 1 (2016).

[54] P. Minkowski, $\mu \rightarrow e \gamma$ at a rate of one out of $10^{9}$ muon decays?, Phys. Lett. 67B, 421 (1977).

[55] T. Yanagida, Horizontal gauge symmetry and masses of neutrinos, Conf. Proc. C7902131, 95 (1979).

[56] S. L. Glashow, The future of elementary particle physics, NATO Sci. Ser. B 61, 687 (1980).

[57] M. Gell-Mann, P. Ramond, and R. Slansky, Complex spinors and unified theories, Conf. Proc. C790927, 315 (1979).

[58] R. N. Mohapatra and G. Senjanovic, Neutrino Mass and Spontaneous Parity Nonconservation, Phys. Rev. Lett. 44, 912 (1980).

[59] J. Schechter and J. W. F. Valle, Neutrino masses in $\mathrm{SU}(2) \times$ U(1) theories, Phys. Rev. D 22, 2227 (1980).

[60] R. N. Mohapatra and J. C. Pati, Left-right gauge symmetry and an isoconjugate model of $C P$ violation, Phys. Rev. D 11, 566 (1975).

[61] R. N. Mohapatra and J. C. Pati, A natural left-right symmetry, Phys. Rev. D 11, 2558 (1975).

[62] G. Senjanovic and R. N. Mohapatra, Exact left-right symmetry and spontaneous violation of parity, Phys. Rev. D 12, 1502 (1975).

[63] R. N. Mohapatra and G. Senjanovic, Neutrino masses and mixings in gauge models with spontaneous parity violation, Phys. Rev. D 23, 165 (1981).

[64] H. Baer, T. Barklow, K. Fujii, Y. Gao, A. Hoang, S. Kanemura, J. List, H. E. Logan, A. Nomerotski, M. Perelstein et al., The international linear collider technical design report-Volume 2: Physics, arXiv:1306.6352.

[65] M. Bicer et al. (TLEP Design Study Working Group Collaboration), First look at the physics case of TLEP, J. High Energy Phys. 01 (2014) 164.

[66] M. Ahmad et al., CEPC-SPPC Preliminary Conceptual Design Report. 1. Physics and Detector (2015), http:// cepc.ihep.ac.cn/preCDR/main_preCDR.pdf.
[67] S. Riemann, Fermion Pair Production at a Linear Collider: A Sensitive Tool for New Physics Searches (2001), http://flc .desy.de/lcnotes/noteslist/index_eng.html.

[68] M. Aaboud et al. (ATLAS Collaboration), Search for Low-Mass Dijet Resonances Using Trigger-Level Jets with the ATLAS Detector in $p p$ Collisions at $\sqrt{s}=13 \mathrm{TeV}$, Phys. Rev. Lett. 121, 081801 (2018).

[69] A. M. Sirunyan et al. (CMS Collaboration), Search for narrow and broad dijet resonances in proton-proton collisions at $\sqrt{s}=13 \mathrm{TeV}$ and constraints on dark matter mediators and other new particles, J. High Energy Phys. 08 (2018) 130.

[70] J. L. Hewett and T. G. Rizzo, Low-energy phenomenology of superstring inspired E(6) models, Phys. Rep. 183, 193 (1989).

[71] C. D. Carone and H. Murayama, Possible Light U(1) Gauge Boson Coupled to Baryon Number, Phys. Rev. Lett. 74, 3122 (1995).

[72] Y. Okada and L. Panizzi, LHC signatures of vector-like quarks, Adv. High Energy Phys. 2013, 1 (2013).

[73] M. E. Peskin and T. Takeuchi, A New Constraint on a Strongly Interacting Higgs Sector, Phys. Rev. Lett. 65, 964 (1990).

[74] M.E. Peskin and T. Takeuchi, Estimation of oblique electroweak corrections, Phys. Rev. D 46, 381 (1992).

[75] L. Lavoura and J. P. Silva, The oblique corrections from vector-like singlet and doublet quarks, Phys. Rev. D 47, 2046 (1993).

[76] C. H. Kom and W. Rodejohann, Four-jet final state in samesign lepton colliders and neutrinoless double beta decay mechanisms, Phys. Rev. D 85, 015013 (2012).

[77] W. Rodejohann, Inverse neutrino-less double beta decay revisited: Neutrinos, Higgs triplets and a muon collider, Phys. Rev. D 81, 114001 (2010).

[78] A. de Gouvêa, W.-C. Huang, and J. Jenkins, Pseudo-Dirac neutrinos in the new Standard Model, Phys. Rev. D 80, 073007 (2009).

[79] A. Donini, P. Hernandez, J. Lopez-Pavon, and M. Maltoni, Minimal models with light sterile neutrinos, J. High Energy Phys. 07 (2011) 105. 